\title{
IMPROVING HYBRID SOLAR CELLS: OVERCOMING CHARGE EXTRACTION ISSUES IN BULK MIXTURES OF POLYTHIOPHENES AND ZINC OXIDE NANOSTRUCTURES
}

\author{
A Thesis \\ presented to \\ the Faculty of California Polytechnic State University, \\ San Luis Obispo
}

\author{
In Partial Fulfillment \\ of the Requirements for the Degree \\ Master of Science in Polymers and Coatings
}

by

Grant Olson

June 2014 
(C) 2014

Grant Olson

ALL RIGHTS RESERVED 
TITLE:

AUTHOR:

DATE SUBMITTED:

COMMITTEE CHAIR:

COMMITTEE MEMBER:

COMMITTEE MEMBER
Improving Hybrid Solar Cells: Overcoming Charge Extraction Issues In Bulk Mixtures Of Polythiophenes And Zinc Oxide Nanostructures

Grant Olson

June 2014

Shanju Zhang, PhD

Assistant Professor of Chemistry

Raymond Fernando, PhD Professor of Chemistry, Director of Polymers and Coatings Program

Robert Echols, PhD Professor of Physics 


\begin{abstract}
Improving Hybrid Solar Cells: Overcoming Charge Extraction Issues in Bulk Mixtures of Polythiophenes and Zinc Oxide Nanostructures
\end{abstract}

\title{
Grant Olson
}

Organic photovoltaics (OPVs) have received a great deal of focus in recent years as a possible alternative to expensive silicon based solar technology. Current challenges for organic photovoltaics are centered around improving their lifetimes and increasing their power conversion efficiencies. One approach to improving the lifetime of such devices has been the inclusion of inorganic metal oxide layers, but interaction between the metal oxides and common conjugated polymers is not favorable. Here we present two methods by which the interactions between polythiophenes and nanostructured $\mathrm{ZnO}$ can be made to be more favorable. Using the first method, direct side on attachment of polythiophene to $\mathrm{ZnO}$ nanowires via chemical grafting, we demonstrate chemical linkage between the polymer and $\mathrm{ZnO}$ phases. The attachment was confirmed to affect the morphological properties of the polymer layer as well, inducing highly ordered regions of the polymer at the $\mathrm{ZnO}$ surface via chemical attachment and physical adsorption. Using the second method to improve polythiophene $\mathrm{ZnO}$ interactions, we have functionalized $\mathrm{ZnO}$ nanowires with organic molecules that favorably interact with conjugated polymer and organic solvents. Photovoltaic devices were made using a blended active layer of functionalized $\mathrm{ZnO}$ 
nanowires and $\mathrm{P} 3 \mathrm{HT}$. Electrical analysis of the resultant devices concluded that the devices were functional photovoltaic cells and isolated the dominant loss mechanisms for further device improvement. 


\section{ACKNOWLEDGMENTS}

I would like to first thank my Advisor, Dr. Shanju Zhang, for his excellent guidance and assistance in the pursuit of my degree. His knowledge and willingness to work with students has been a foundation for my research, and I would have been unable to come as far as I have without his help.

I would like to thank Dr. Robert Echols, who originally set me on the path of researching organic photovoltaics. His commitment to undergraduate student involvement in research fostered my passion for the solar energy field, and his guidance has proved invaluable in developing my own methods to solar cell fabrication and characterization.

I would also like to thank Dr. Ray Fernando, who was instrumental in getting me involved with the Polymers and Coatings program, and made it possible for me to switch graduate programs when it became clear that my focus was closely aligned with current areas of research.

I would like to thank my colleagues Taylor Wagner, Neil Redeker, and Grace Luo, as well as the rest of the members Dr. Zhang's research group for their support and insight.

I would like to thank Buddy Bump for his continued help with solar device fabrication and characterization.

Additionally, I would like to thank the Polymers and Coatings Program as well as the Chemistry and Biochemistry Department department for their support of me and this project. I would also like to thank the Materials Engineering Department for use of their instrumentation and their technical support 
This project was made possible by funding from the National Science Foundation under CMMI-1345138. 
LIST OF FIGURES $\quad$ ix

INTRODUCTION

BACKGROUND 4

$\begin{array}{ll}\text { ORGANIC PHOTOVOLTAICS } & 7\end{array}$

HYBRID PHOTOVOLTAICS 19

METHODS AND MATERIALS 26

PREPARATION OF ZINC OXIDE NANOWIRES 26

CHARACTERIZATION AND ANALYSIS 28

FOURIER TRANSFORM INFRARED SPECTROSCOPY 28

THERMAL GRAVIMETRIC ANALYSIS 28

UV-VISIBLE ABSORPTION SPECTROSCOPY 29

UV-VISIBLE FLUORESCENCE SPECTROSCOPY 29

X-RAY DIFFRACTION 30

TRANSMISSION ELECTRON MICROSCOPY 30

DEVICE FABRICATION AND CHARACTERIZATION 31

CURRENT DENSITY-VOLTAGE MEASUREMENT 32

OPTICAL DENSITY SPECTROSCOPY 33

RESULTS AND DISCUSSION 34

ZINC OXIDE NANOCOMPOSITE CHARACTERIZATION 34

FUNCTIONALIZED ZNO NANOSTRUCTURES FOR HYBRID SOLAR CELLS

CONCLUSIONS

$\begin{array}{ll}\text { REFERENCES } & 60\end{array}$ 


\section{LIST OF FIGURES}

Figure 1 - Cost of solar electricity broken down by component pricing

Figure 2 - National renewable energy plot of solar cell efficiencies over the past four decades. Current record efficiencies for each device type are listed in the righthand margin

Figure 3 - Diagram of electronic bands of conductors (left), semiconductors (middle) and insulators (right). The grey lines through the bands indicate the presence of electrons; electrons in the conduction band are a characteristic element of conductive materials. The dotted line (Fermi energy) represents the equilibrium energy of electrons in the material

Figure 4 - Energy band diagram for electrical contact between material with dissimilar Fermi energies. This junction forms the basis of electronic devices such as diodes, transistors, LEDs, and solar cells

Figure 5 - The valence and conduction bands of a direct band gap material (left) and an indirect band gap material (right). Direct band gap transitions occur directly from the top of the valence band into the bottom of the conduction band. Indirect band gap materials require a higher energy photon to excite, or require a phonon interaction (red arrow) in addition to the photon excitation when transitioning

Figure 6 - Common conducting polymers, polyphenylene (left), polyphenylvinylene (middle) and polythiophene (right)

Figure 7 - Poly(3-hexylthiophene) and Phenyl-C61-butyric acid methyl ester, two of the most common components in conjugated polymer solar cells

Figure 8 - Energy levels of P3HT:PCBM solar cell. Excitons form when photons excite electrons from the LUMO to the HUMO level of P3HT

Figure 9 - Exciton dissociation range in P3HT:PCBM. Light absorbed more than $10 \mathrm{~nm}$ from an interface cannot be converted into electricity

Figure 10 - Comb structure (left) and bulk heterojunction (right) of dissimilar donor-acceptor materials in polymer photovoltaics

Figure 11 - Example Voltage-Current Density curve for solar cell testing

Figure 12 - Effect of polymer conformation on molecular band energies

Figure 13 - The structure of a hybrid bulk heterojunction device

Figure 14 - TEM image of P3HT and pristine $\mathrm{ZnO}$ nanowires, displaying no interaction

Figure 15 - TPP and DDT functionalization of $\mathrm{ZnO}$ nanowires to improve interactions with solvent and $\mathrm{P} 3 \mathrm{HT}$ 
Figure 16 - Scheme for functionalization of $\mathrm{ZnO}$ nanowires via carboxylated polythiophenes. The polymer spontaneously reacts with the nanowire in solution, forming a side on attachment. After sufficient reaction time the $\mathrm{ZnO}$ is completely coated and forms a core-shell structure

Figure 17 - From left Poly(3-carboxy-hexyl-thiophene), Poly(3-carboxy-pentyl-thiophene), Poly(3-carboxy-butyl-thiophene), and Poly(3-carboxy-propyl-thiophene).

Figure 18 - Device Fabrication steps. A) Begin with prestencilled ITO glass.

B) Apply and wipe away PEDOT:PSS. C) Apply and wipe away active layer.

D) Evaporate metal electrodes

Figure 19 - FTIR spectra of carboxylated polythiophene and $\mathrm{ZnO}$ polythiophene nanocomposites synthesized in pyridine.

Figure 20 - Thermal gravimetric analysis of $\mathrm{P} 3 \mathrm{C}(\mathrm{n}) \mathrm{T}$ nanocomposites

Figure 21 - TGA derivatives comparing composite decompositions with polymer decompositions

Figure 22 - TEM imaging of ZnO polythiophene nanocomposite. Rods fabricated exhibit a variance in length, but are relatively homogenous in diameter (top left). The polymer shell of the nanocomposite is bonded to the nanowire but can interact with polymer from other, similarly bonded nanowires (top right). The shell of the polymer is believed to be capable of forming monolayers given lower loadings, while the excess polymer can physically adsorb onto the surface of the modified nanowire (bottom left). Excessive polymer loading can lead to clumps of functionalized nanowires that are very difficult to disperse (bottom right).

Figure 23 - TEM images of high loading $\mathrm{ZnO}$ polythiophene nanocomposites

Figure 24 - UV-Vis of carboxylated polythiophenes in DMSO

Figure 25 - UV-Vis absorbance spectroscopy of $\mathrm{ZnO}$ nanowire polythiophene composites in DMSO

Figure 26 - UV-vis absorbance spectroscopy comparing polymer and composite materials in DMSO

Figure 27 - UV-Vis absorbance spectroscopy of the polythiophene shell of nanocomposite in DMSO

Figure 28 - Fluorescence spectra for carboxylated polythiophene and polymer nanocomposite in DMSO excited at $450 \mathrm{~nm}$.

Figure 29 - Fluorescence spectra for carboxylated polythiophene in DMSO, excited at 450nm

Figure 30 - Fluorescence spectra for carboxylated polythiophenes and nanocomposites with $\mathrm{ZnO}$ nanowires in DMSO, excited at 450nm 
Figure 31 - Fluorescence spectra for polymer composites in DMSO, excited at 450nm

Figure 32 - Open circuit voltage plot of surface functionalized $\mathrm{ZnO}: \mathrm{P} 3 \mathrm{HT}$ bulk heterojunction hybrid devices

Figure 33 - Short circuit current plot of surface functionalized $\mathrm{ZnO}: \mathrm{P} 3 \mathrm{HT}$ bulk heterojunction hybrid devices

Figure 34 - Typical device dark curve from the 5:5 functionalized $\mathrm{ZnO}: \mathrm{P} 3 \mathrm{HT}$ groups

Figure 35 - Power Conversion efficiency plot of surface functionalized ZnO:P3HT bulk heterojunction hybrid devices

Figure 36 - Optical density measurements for solid film absorbances of surface functionalized $\mathrm{ZnO}: \mathrm{P} 3 \mathrm{HT}$ bulk heterojunction hybrid devices

Figure 37 - Fill factor plot of surface functionalized $\mathrm{ZnO}: \mathrm{P} 3 \mathrm{HT}$ bulk heterojunction hybrid devices 


\section{INTRODUCTION}

It is projected that the electricity required by the human race in the year 2030 will reach 16.9 TW, a number that is dwarfed by the 580TW of accessible solar power incident on Earth's surface. ${ }^{1}$ While the US currently generates $13 \%$ of it's electricity using renewable sources, only 2\% of that comes from solar electrical generation. ${ }^{2}$ Given that the energy is abundant, clean, and the generation easy to maintain once the infrastructure is in place, the only thing that prevents solar from becoming a primary energy source is the cost associated with it. ${ }^{3}$ In 2011 the Department of Energy released a report that stated solar power generation would become competitive with conventional forms of electricity generation if the cost of generated electricity could be reduced to $\$ 0.06$ per kWh. ${ }^{4}$ The industry has been advancing rapidly to reach this goal, see Figure 1, but the standard silicon technology that has been the mainstay of commercial solar may not be able to push all the way to cost competitive levels. Silicon, for all it's advantages, has several material drawbacks that are difficult to overcome, making the development of new solar technologies a desirable. On such region is in organic photovoltaics, which utilize a carbon based active material instead of silicon. While the challenges presented in working with carbon based materials are significant, the very real possibility of a cost effective solar generation system is driving heavy research into the field of organic solar. 
The Falling Price of Utility-Scale Solar Photovoltaic (PV) Projects

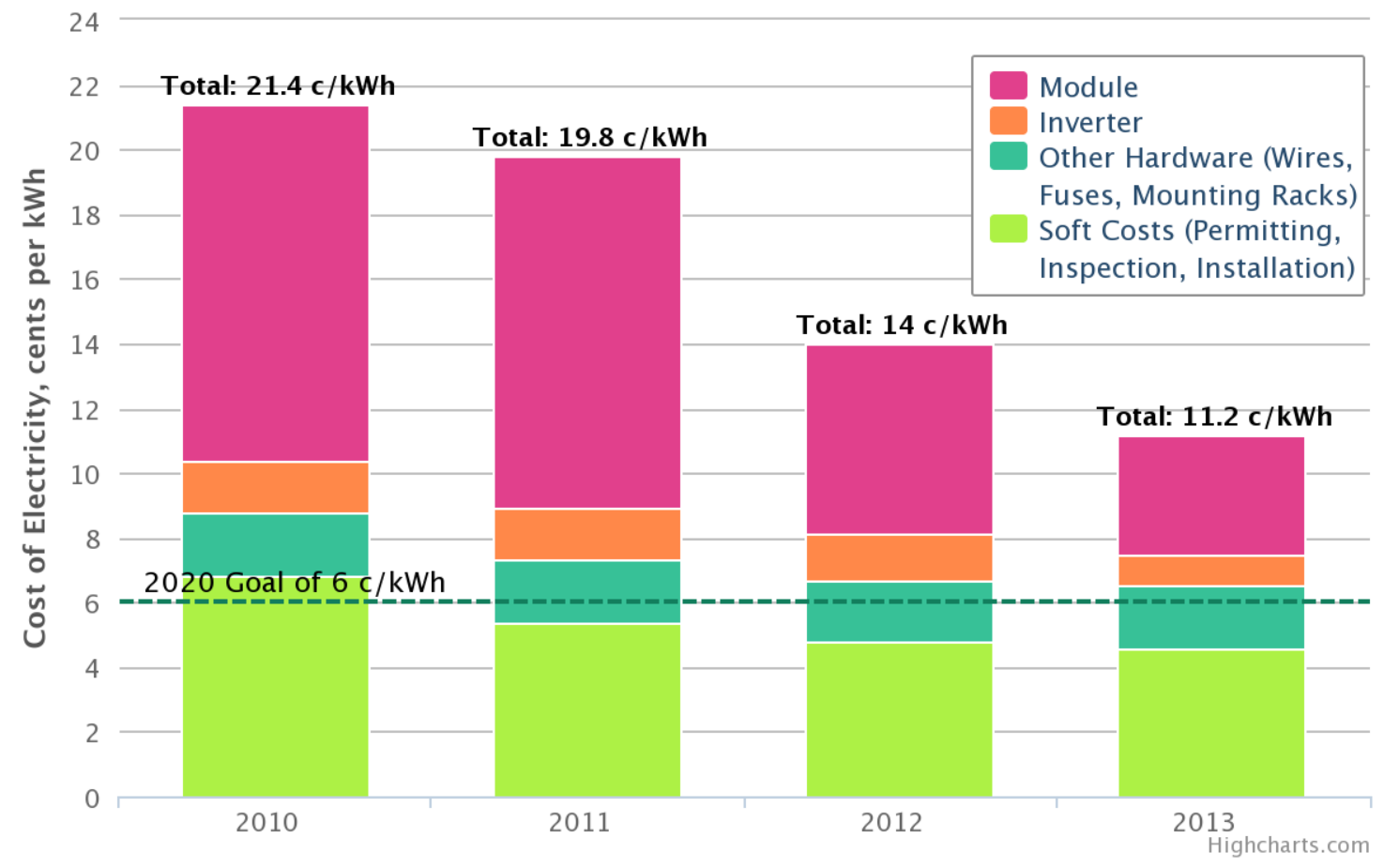

Figure 1 - Cost of solar electricity broken down by component pricing. ${ }^{5}$

Utilizing the energy of the sun predates the existence of our species, but direct harvesting of solar energy to do electrical work can be traced back to the last few hundred years. The first recorded photovoltaic device was observed in 1876, when an illuminated interface between platinum and selenium created an induced voltage ${ }^{6}$ Einstein's paper on the photoelectric effect in $1905^{7}$ provided the theoretical backdrop for the development of the modern solar cell almost fifty years later. Bell labs, in 1954, developed the first modern solar cell utilizing silicon semiconductor technology. ${ }^{8}$ The first cells were $4 \%$ power conversion efficient, but current technologies can create solar devices with upwards of $40 \%$ power conversion efficiency. ${ }^{9}$ The National Renewable Energy Laboratory (NREL) periodically publishes a report detailing the record values reported and 
confirmed for various solar technologies, shown in Figure 2. The last 15 years in particular has seen a rise of new technology exploding upwards in efficiency, driving the industry to the point where it can become cost competitive with other forms of electricity generation.

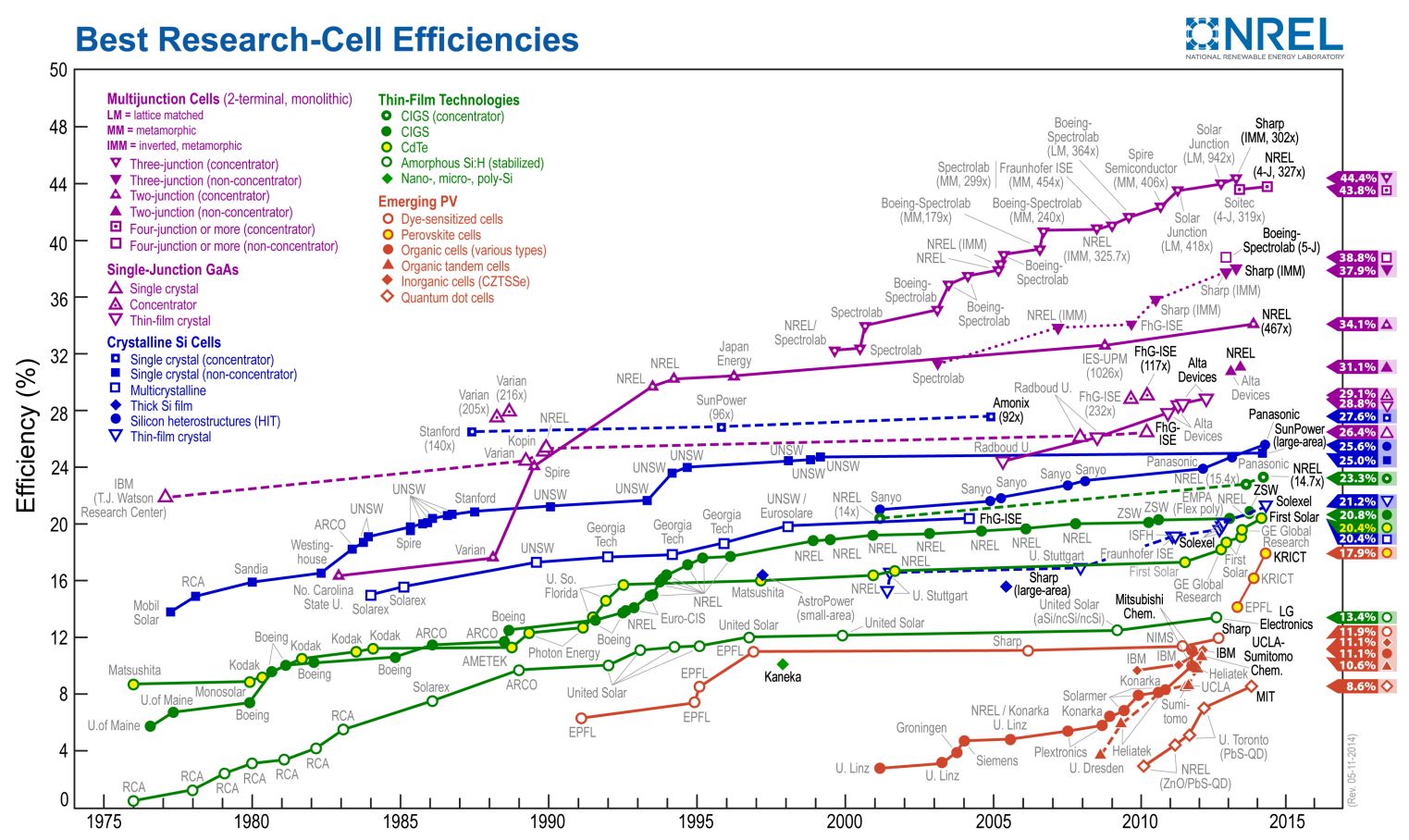

Figure 2 - National renewable energy plot of solar cell efficiencies over the past four decades. Current record efficiencies for each device type are listed in the righthand margin ${ }^{10}$ 


\section{BACKGROUND}

From the standpoint of solid state physics, the conductivity of materials can be attributed to partially filled electron energy bands. ${ }^{11}$ In conductors, the equilibrium chemical energy of the electrons, referred to as the Fermi energy, resides in a material's electron band, resulting in a partially filled band state referred to as the conduction band. Semi-conductors and insulators have a Fermi level that lies between band states, resulting in a band below the Fermi energy that is completely filled, and a band above it that is completely evacuated, shown in Figure 3. Physically, thermal energy allows electrons to excite across the band gap between the valence and conduction bands, but the probability of such events are dependent on the available thermal energy and impeded by larger band gaps. The difference between insulators and semiconductors lies in the size of the gap between their conduction and valence bands.

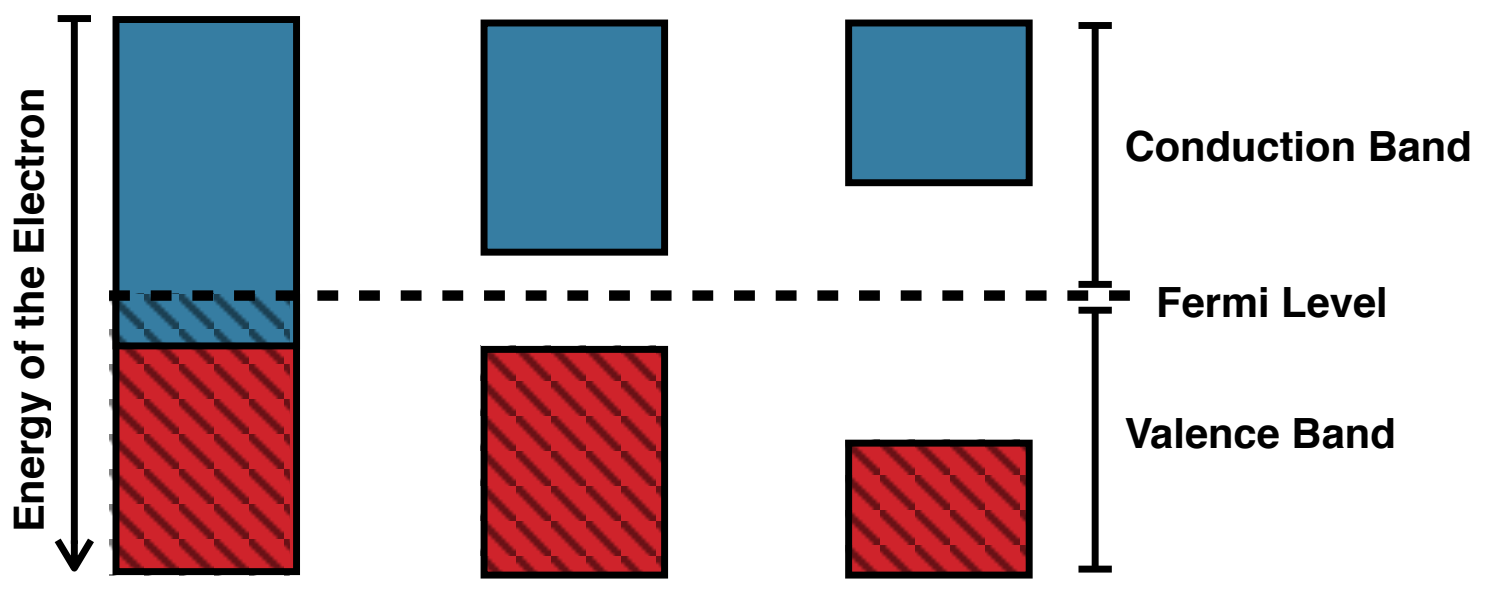

Figure 3 - Diagram of electronic bands of conductors (left), semiconductors (middle) and insulators (right). The grey lines through the bands indicate the presence of electrons; electrons in the conduction band are a characteristic element of conductive materials. The dotted line (Fermi energy) represents the equilibrium energy of electrons in the material 
The energy that the Fermi level represents will equilibrate at the point of contact between two materials who's Fermi levels differ. ${ }^{12}$ This band equilibration changes the effective energy levels of the conduction and valence bands of the two materials, which is important for applications where the interactions between the valence and conduction bands drives the electrical properties of the device, and is shown in Figure 4.
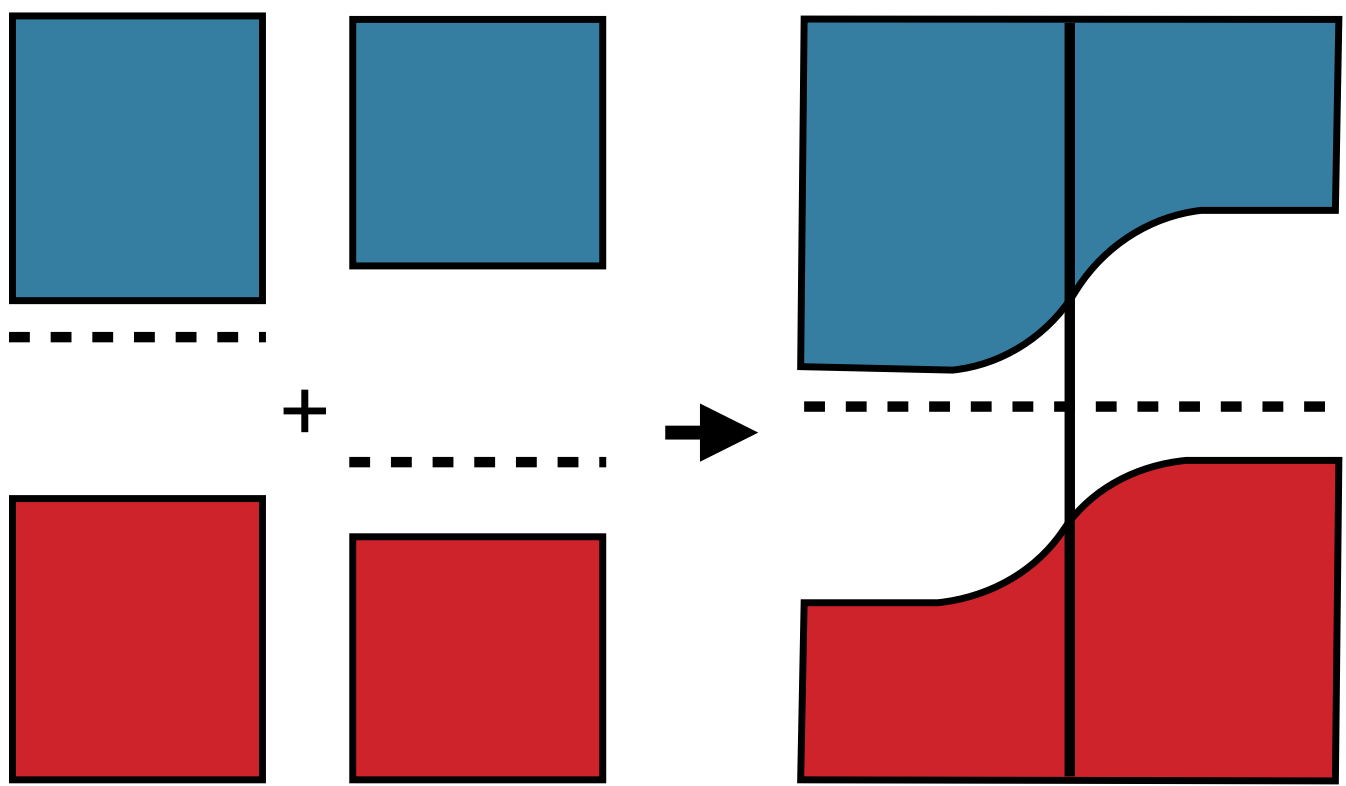

\section{$+$}

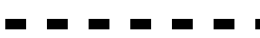

n-type

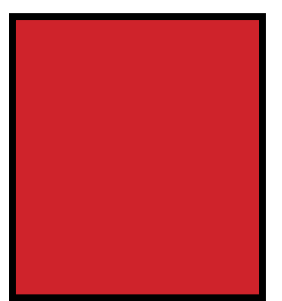

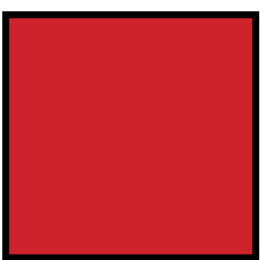

p-type

Figure 4 - Energy band diagram for electrical contact between material with dissimilar Fermi energies. This junction forms the basis of electronic devices such as diodes, transistors, LEDs, and solar cells

At the most fundamental level, photovoltaic technology is dependent on creating free charges in the presence of an electric field. In conventional inorganic semiconductor devices, like silicon or germanium, the free charges are generated by a synthetic interface between n-type and p-type doped regions. Sufficiently energetic light that is absorbed in the $n$-type material causes an 
electron in the valence band to excite into the conduction band, leaving behind a region of positive charge referred to as a hole. The different charges of the electron and the hole cause them to attract each other and they are electrostatically bound together, making an uncharged exciton. However, because of the alignment between the conduction band of the n-type material and the valence band of the p-type material, the electron-hole pairs that are sufficiently close to the interface between the two phases will travel to the interface and dissociate, with the electron transitioning into the valence band of the p-type material. At this point the electron and the hole are no longer bound, and can be affected by electric fields to induce motion. Without external help, the buildup of charge at the interface between the $n$-type and $p$-type materials will create an electric field that will push charges away, but the process of charge extraction can be augmented through the selection of electrodes. The work function difference, which is the energy difference in the Fermi levels between the conduction bands of two metals, will create an inherent electric field. ${ }^{13}$ By selecting two electrodes with different Fermi energies, an induced electric field can push free charges towards the electrodes in a favorable way.

Silicon based solar cells, which are currently the most widely implemented photovoltaic technology, bear certain material disadvantages. As shown in Figure 5, valence and conduction bands of silicon are not aligned with respect to the wave number, which means that a transition between the valence and conduction bands requires either greatly more energy than the band gap would indicate, or an additional phonon interaction to change the electron's momentum 
to match the band momentum. This phenomena is referred as an indirect band gap, and has the notable effect of decreasing the absorbance of the material in question. ${ }^{14}$ Because of this, silicon will always have a high material cost, and the thick layers required also prevent silicon from being usable as a flexible solar material.

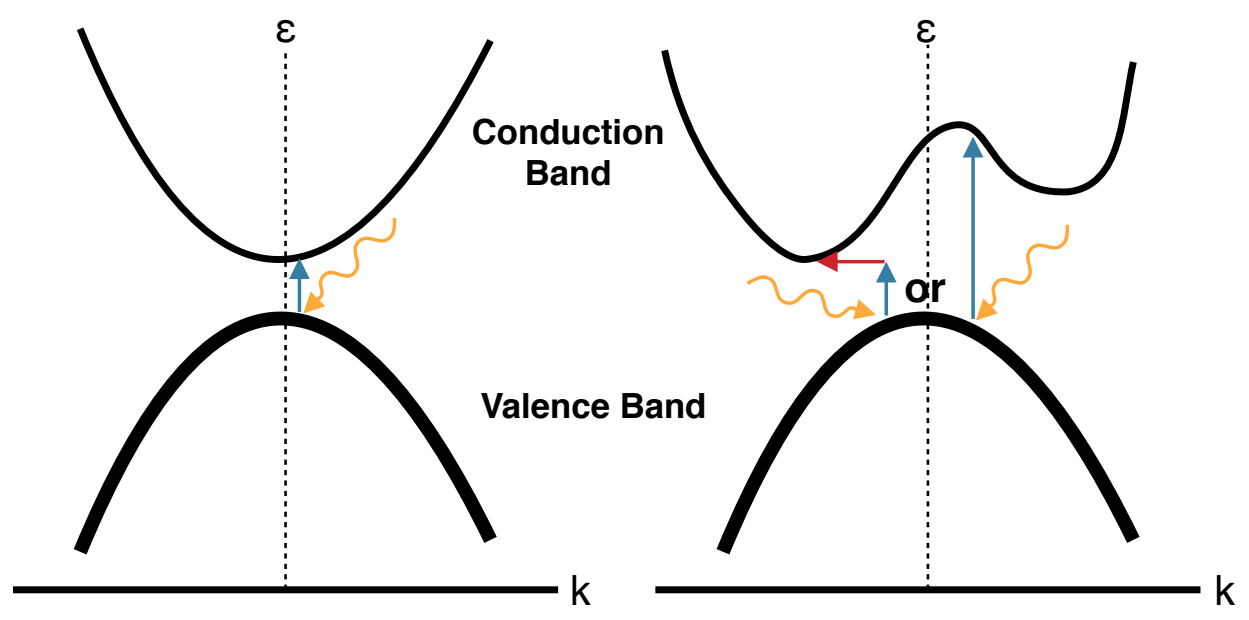

Figure 5 - The valence and conduction bands of a direct band gap material (left) and an indirect band gap material (right). Direct band gap transitions occur directly from the top of the valence band into the bottom of the conduction band. Indirect band gap materials require a higher energy photon to excite, or require a phonon interaction (red arrow) in addition to the photon excitation when transitioning

\section{ORGANIC PHOTOVOLTAICS}

Conductive polymers were discovered by a research group from the University of Pennsylvania in $1977 . .^{15}$ The materials used were halogen doped polyacetylenes, which showed drastically higher conductivities than any other organic polymer previously investigated. While polyacetylene has not been developed as a widely used conducting polymer, the fundamental aspect that supplies it with its conductive qualities is similar to the polymers that are being 
used today; most notably the conjugated backbone, containing carbons that are double bonded to each other. Whereas the conductivity of metals can be thought of as freedom of movement in the unbound electron pairs of the molecular orbitals, the conductivity of conjugated polymers stems from the exceptionally diffuse electron cloud associated with carbon double bonds. ${ }^{16}$ A backbone containing a high degree of conjugation is likely to manifest conductive qualities. Some commonly used conductive polymers are shown below in Figure 6 , all of which have a highly conjugated backbones.

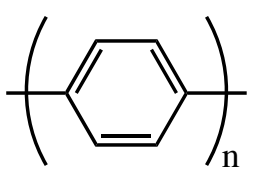<smiles>Cc1ccc(/C=C/C(C)C)cc1</smiles>

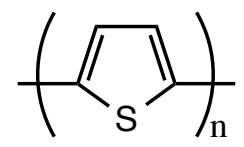

Figure 6 - Common conducting polymers, polyphenylene (left), polyphenylvinylene (middle) and polythiophene (right)

The photovoltaic mechanism of the polymer solar cell is similar in nature to the mechanism of the silicon solar cell. Two phases exist, one which will absorb the light and excite an electron into a higher energy band, leaving behind a hole, while the other phase will accept the electron from the exciton, freeing the charges for extraction. The materials used to create the two phases are, however, dissimilar in polymer

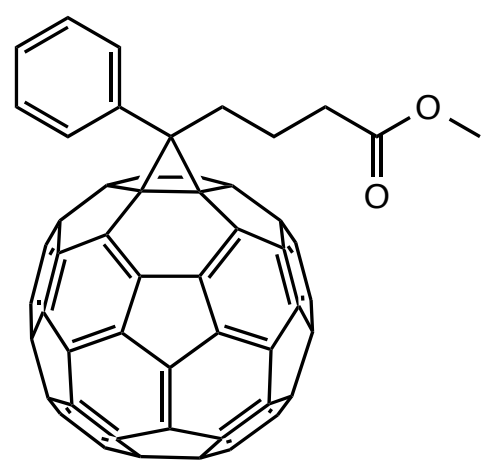

Figure 7 - Poly(3-hexylthiophene) and Phenyl-C61-butyric acid methyl ester, two of the most common components in conjugated polymer solar cells

solar cells; instead of using one material which is doped to change the material's 
Fermi energies, a donor and acceptor material are used which have energy levels that are already functional. A common choice of donor is Poly(3hexylthiophene), or $\mathrm{P} 3 \mathrm{HT}$, and a common electron acceptor is Phenyl-C61butyric acid methyl ester, or PCBM, both shown in Figure 7. PCBM is not actually a polymer, but is instead a conjugated carbon sphere with an added tail for solubility in organic solvents. These materials have molecular orbitals, which take the place of valence and conduction bands in discussion of the excitation energies necessary for charge generation, the total energies of which are shown in Figure 8. The energy gap between the highest occupied molecular orbital (HOMO) and the lowest unoccupied molecular orbital (LUMO) of the P3HT defines the wavelengths of light over which the solar device will be able to meaningfully generate charge. The LUMO level of the PCBM must be below the LUMO of the P3HT to allow dissociation of excitons.

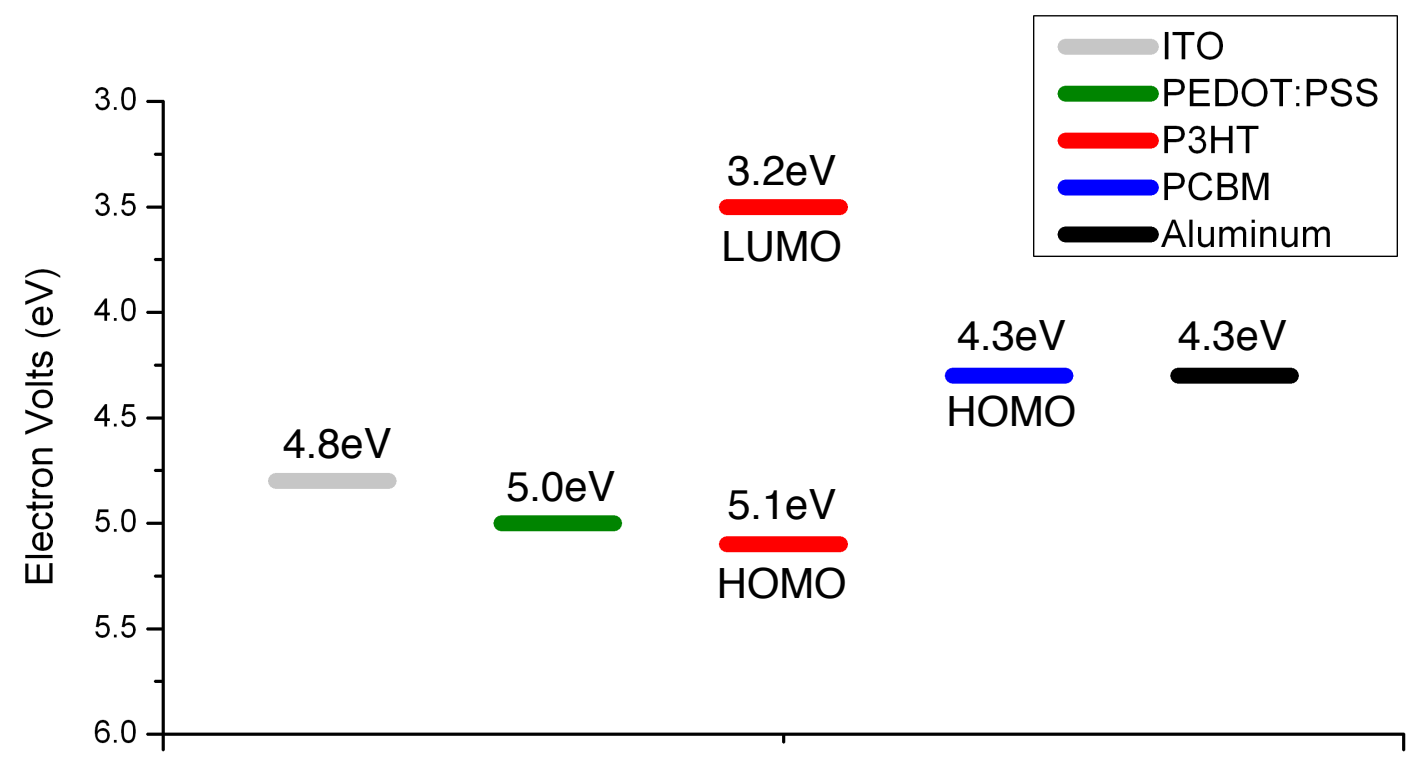

Figure 8 - Energy levels of P3HT:PCBM solar cell. Excitons form when photons excite electrons from the LUMO to the HUMO level of P3HT.17,18 
The inherent advantage in using conducting polymers as compared to their inorganic predecessors comes down to material cost, processability and flexibility. Because silicon maintains an indirect band gap, devices must be several microns thick in order to absorb a large enough quantity of light to effectively generate electricity. Polymeric materials, such as polythiophenes, have a direct band gap, which means they have high absorbance, requiring a layer only a few hundred nanometers thick to absorb most of the light incident upon a device. ${ }^{19}$ Additionally, while silicon's fabrication process is labor and energy intensive, photovoltaic polymers can be dissolved in organic solvents and applied via common coating processes such as screen printing or roll-to-roll printing..$^{20}$ The up-scaleability of polymer based solar far outstrips silicon technology. Finally, owing in part to its required thickness and in part to its mechanical properties, silicon solar panels are rigid and fragile to mechanical stresses. Organic solar devices have been developed that are highly flexible and can undergo enormous repeated strains with minimal loss to device performance..$^{21}$

The challenges currently facing organic solar technology take the form low power conversion efficiency and rapid device degradation in real world conditions. The average silicon solar panel is about $20 \%$ efficient, whereas the best organic solar cell made in a research laboratory is $11.1 \%$ efficient. ${ }^{22}$ Additionally, while silicon solar panels have effective lifetimes that exceed 20 years, the polymers used in plastic solar cells are vulnerable to photo assisted oxidation, as well as more conventional oxidation reactions in the presence of 
oxygen and water. Because of this, fabrication of the cells must take place in an inert atmosphere and the packaging of the cells must prevent water and oxygen from penetrating the active layer. Research has shown that incorporation of metal oxide materials in close proximity with the active layers can protect the polymer components to a certain degree, extending the device lifetimes. ${ }^{23}$

One significant difference between the classical silicon solar cell and organic photovoltaics are the exciton lifetimes, or the time between electron excitation and its decay back into a lower energy state. In P3HT the exciton lifetime is $\sim 15 \mathrm{fs}$, which corresponds to a travel distance of $10 \mathrm{~nm},{ }^{25}$ so any excitons that are created

more than $10 \mathrm{~nm}$ from a material interface will recombine instead of separating, see Figure 9.

This will result in the energy being lost as heat instead of being turned into electricity.

The ideal device structure would be one in which the donor (P3HT) material phases are no more than $20 \mathrm{~nm}$ across by their smallest dimension, and with

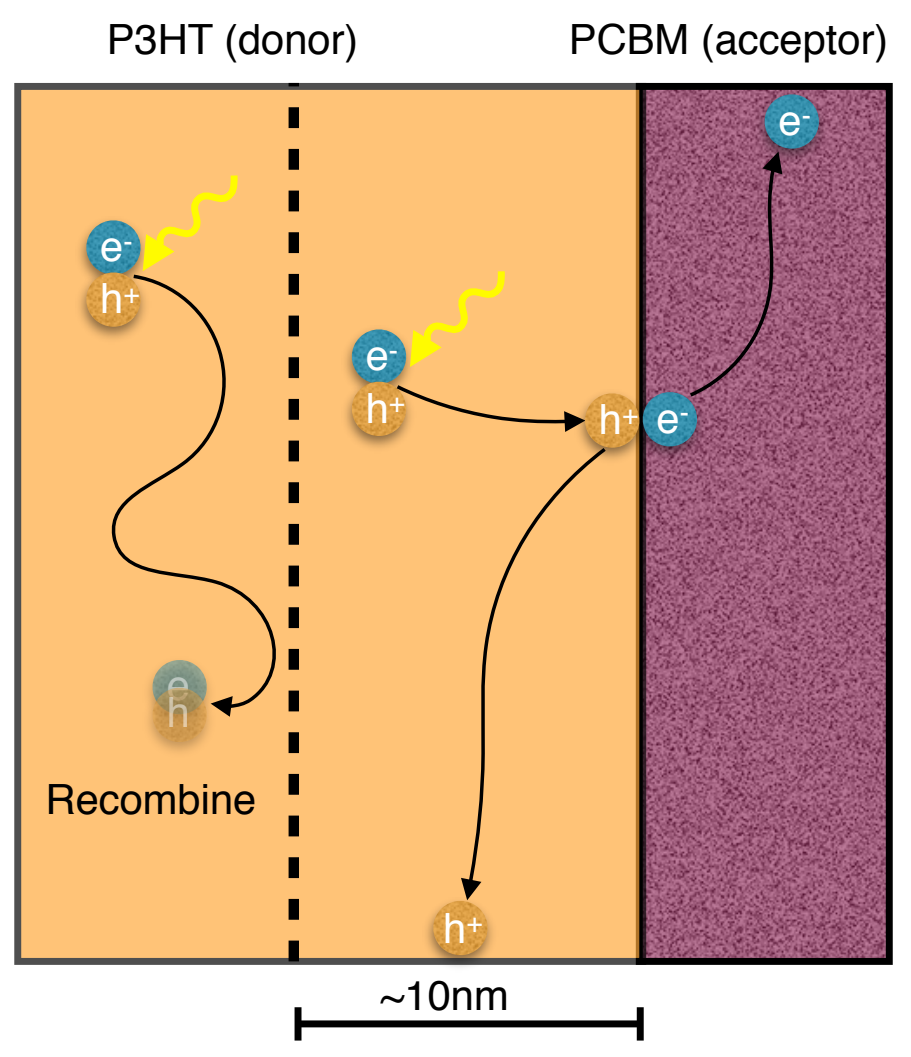

Figure 9 - Exciton dissociation range in P3HT:PCBM. Light absorbed more than $10 \mathrm{~nm}$ from an interface cannot be converted into electricity 
continuous interaction between the donor and the acceptor phase. An example of an ideal heterojunction structure would be the comb, shown in Figure 10, where the donor and the acceptor material are inter-dispersed and their ordered phases are oriented normal to the electrode to minimize the travel distance of the separated charges.

In practice, creating a comb structure on the nanometer scale not a viable method for commercial production. A bulk production method is required to maintain the low cost potential of polymer solar. Self assembly of a bulk heterojunction is an easy solution to this problem, where the donor and acceptor materials are mixed in solution and applied together. ${ }^{26}$ Forming a functional bulk heterojunction requires that the materials self assemble when they are mixed and applied from solution, so the application methods and materials are restricted in this technique. Research has shown that high functioning devices can be fabricated through selection of materials and through careful control of processing conditions. ${ }^{25}$ The bulk synthesis of active material layers does have the disadvantage of lacking direct control over region sizes and distributions.
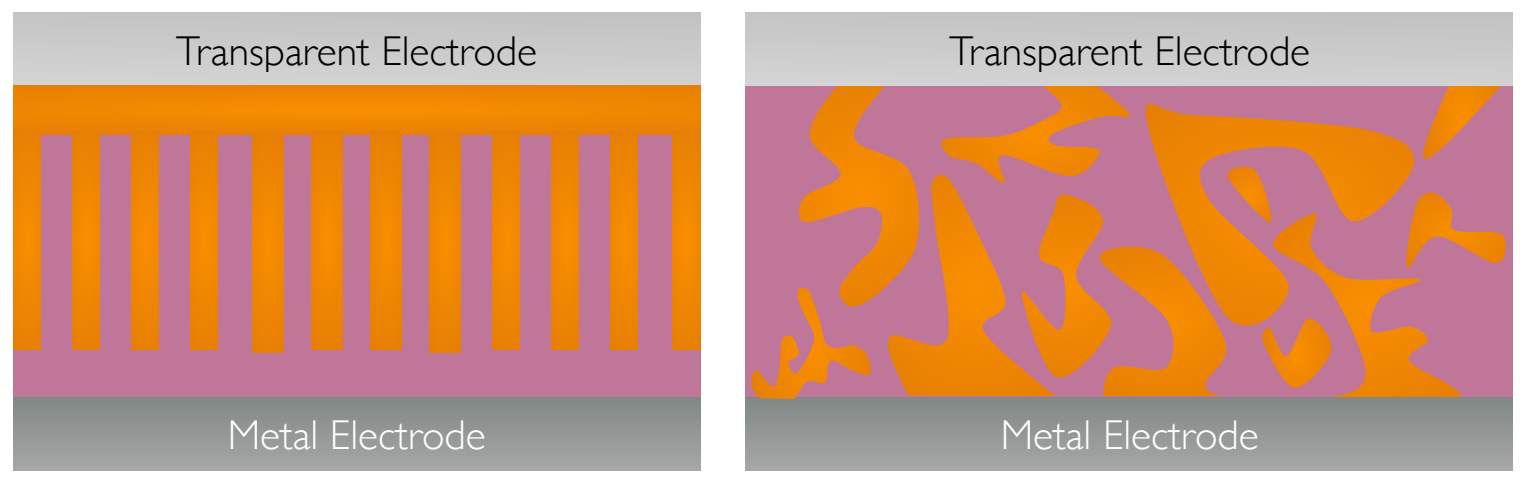

Figure 10 - Comb structure (left) and bulk heterojunction (right) of dissimilar donor-acceptor materials in polymer photovoltaics 
Creating optimal device structures from a self assembling layer can difficult, and because of the small size and relative similarity of the chemical makeup of the two phases, it is often challenging to identify the source of poor device performance when it occurs. Electronic testing of final devices can give some insight into the dominant loss mechanisms. Typical solar cell testing in modern industry involves the use of a variable voltage-current source, which applies a series of known biases to a device and measures the current. ${ }^{27}$ This method is referred to as $\mathrm{JV}$ sweeping, utilizing the symbol $\mathrm{J}$ as opposed to $\mathrm{I}$ as the values are typically reported in current density $\left(\mathrm{A} / \mathrm{m}^{2}\right)$ to eliminate the device size dependence. An example plot of a JV curve is shown in Figure 11, with the general points of interest indicated and labelled. A standard analysis suite for solar devices will measure, from the JV curve, the open circuit voltage, $V_{\mathrm{oc}}$, short circuit current density, $\mathrm{J}_{\mathrm{sc}}$, and the power conversion efficiency, $\eta$, given that the incident light intensity is known. Fill factor, the ratio between the max power generated and the product of the $\mathrm{V}_{\mathrm{oc}}$ and $\mathrm{J}_{\mathrm{sc}}$, is often calculated and used as an indicator of prevalent loss mechanisms. Other useful pieces of information may include the forward pass current, which is the current passed through the device at some designated point in the forward bias regime i.e. $+1 \mathrm{~V}$, and the reverse bias leakage, which similarly is the current passed backwards through the device under a reverse bias i.e. $-1 \mathrm{~V}$. A well performing solar cell will have a JV curve that closely resembles a diode, with little reverse current, and an exponential increase in the forward pass current after some turn-on voltage. The power 
generation region of the device is in quadrant 4 , where the voltage is forward (positive) but the device continues to generate reverse current (negative).

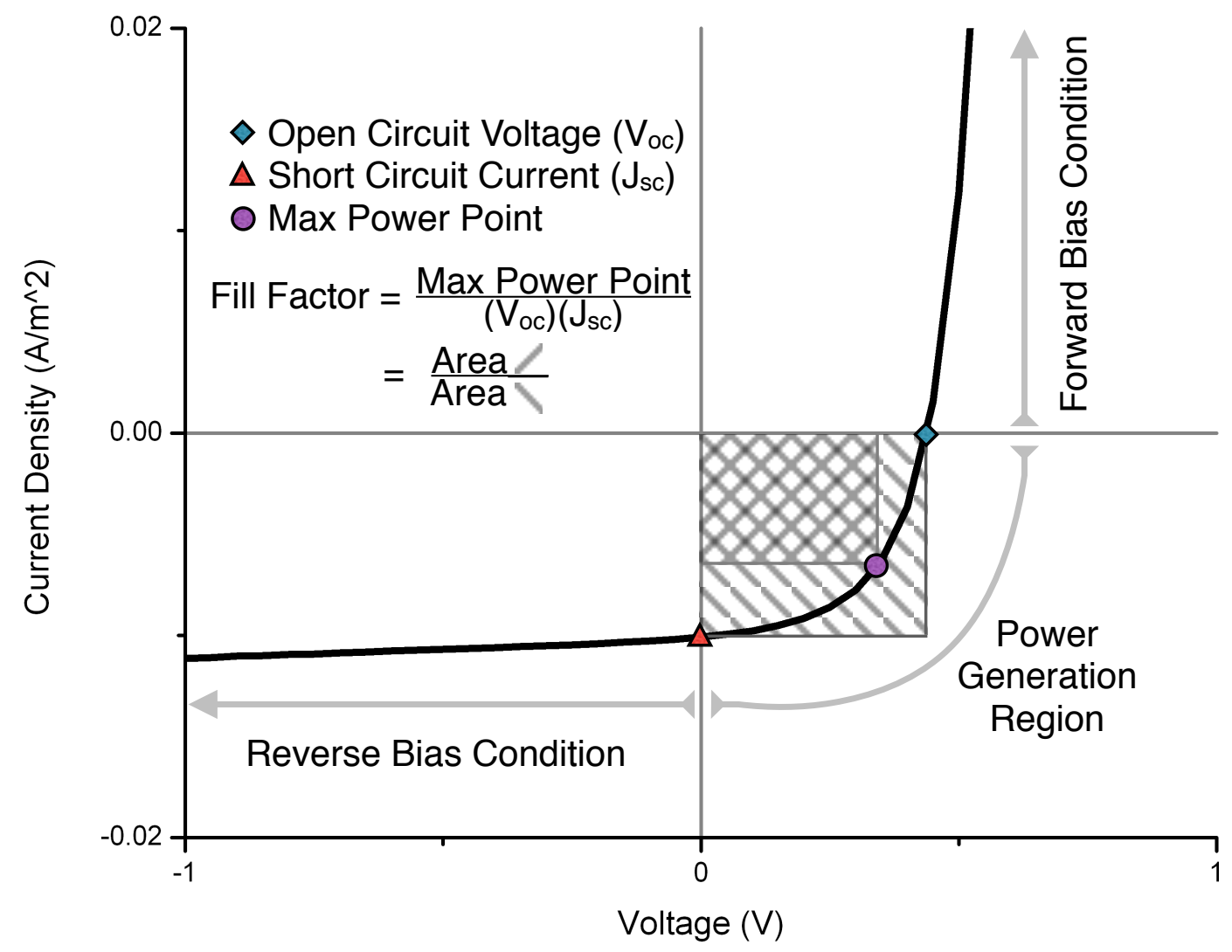

Figure 11 - Example Voltage-Current Density curve for solar cell testing

Common loss mechanisms for electrical energy generation in photovoltaic devices include; recombination, poor charge extraction (trapping), thermalization, leakage, and resistive losses. ${ }^{28,29}$ Each loss mechanism is attributable to one or more physical characteristics within a device, and each tends to have a characteristic electrical signature. This allows us to infer issues with device 
fabrication and active layer morphology from the electrical testing and adjust systems accordingly.

The first loss mechanism, recombination, occurs when excitons are unable to reach or interact with an interface between the donor and the acceptor material before recombining. This is often caused by donor regions that are too large in size, or by non-ideal donor-acceptor interfaces. In both cases, excitons have a hard time reaching an interface to dissociate into free charges.

Electrically, recombination is typically indicated by low $\mathrm{J}_{\mathrm{sc}}$, with comparatively normal $V_{o c}$, unless the interface is so poor as to impede electrical interaction all together. The fill factor is often unaffected by recombination loss; the decrease in current affects the short circuit condition equally with the max power condition. Forward pass currents for devices with this issue are typically quite high, as recombination does nothing to impede the device in forward bias conditions, unless the donor-acceptor interface is very unfavorable. To mitigate this loss mechanism, a device with smaller regions and better donor-acceptor interfaces must be developed. Typically, this is a materials problem, as the regions of bulk heterojunctions are self assembling, and the material interfaces are determined by materials characteristics. However, there are processing techniques that can influence polymer region size and crystallinity, such as thermal anneal, which could be used to deal with this issue.

The second loss mechanism is poor charge extraction, which occurs when charges that have already dissociated encounter resistance while traveling their respective electrodes. Trapping is a special case of this, in which free charges 
are separated into an island of donor or acceptor material, without access to an electrode. Poor charge extraction is attributable to donor and acceptor regions that are too small, donor and acceptor materials that are not sufficiently conductive, and poor interaction between the active layer and electrodes. In JV plots, poor charge extraction leads to low $\mathrm{J}_{\mathrm{sc}}$ and poor fill factors, with relatively untouched $V_{\text {oc. }}$. The forward pass current in devices with poor charge extraction tends to be low, because the tortuous pathways continue to affect current even when it's externally induced. To prevent charge extraction issues, device regions need to be made larger and more conductive, or the interface between the active layer and the electrode needs to be improved. These issues are more easily addressed by post fabrication processing than recombination, because polythiophenes tend to self aggregate via spinodal decomposition when heated, ${ }^{48}$ which means that thermal anneal will increase polymer region size and crystallinity. Also, interactions between the electrode and the active layer can be improved via similar thermal processing.

The third loss mechanism is thermalization, which is impacted by the electrical interactions between the bands of the active materials. This loss actually occurs in two phases. First, when a photon is absorbed that has higher energy than the lowest energy difference between the HOMO and LUMO levels of the donor material, the excess energy is lost as heat. Then, when the generated exciton reaches an interface and dissociates, the energy difference between the bands is also lost to heat. A certain gap between the two LUMO levels is required to induce a favorable transition for the electron, but the 
advantage of improving the number of electron's extracted must be balanced with the amount of energy each of those electrons has left. ${ }^{30}$ In the P3HT:PCBM system, half of the electron's excitation energy is immediately lost to thermalization upon charge dissociation, making the maximum possible voltage that a single layer $\mathrm{P} 3 \mathrm{HT}: \mathrm{PCBM}$ device could ever produce be $<0.8 \mathrm{~V}$, and more typically around $0.5 \mathrm{~V} .{ }^{31}$ Thermalization losses set the voltage maximum of a material system, as such the thermalization losses tend to effect $V_{o c}$ exclusively. It is difficult to impact thermalization of a device without changing the material system. Subtle things can be done to manipulate the energy bands of the donor or the acceptor material, which can reduce some of the losses. P3HT in particular grants a method of controlling thermalization loss because it is a semicrystalline conducting polymer; changes in the size of ordered regions can expand the energy bands of materials like $\mathrm{P} 3 \mathrm{HT} .{ }^{32}$ The details of band manipulation via polymer conformation will be covered in more depth in a later section.

The fourth and fifth loss mechanisms, leakage and resistive loss, have to do primarily with the design of the device and how the active materials interact with the electrodes. The thin film nature of organic electronics make them susceptible to micro shorting, when a conductive pathway is formed between the anode and the cathode. Interstitial layers like PEDOT:PSS, which can be used to improve the work function of device electrodes, are also conductive enough to form leakage pathways if the device is not designed well. Resistive loss within a device could be called the opposite problem; if the conducting pathways within a 
device are not transmitting charge without significant loss, or in even worse cases inconsistent loss, then both performance and the ability to define the device issues will be negatively effected. Leakage within a device is uniquely characterized by a high reverse bias leakage, as the micro shorts or macroscopic leakage pathways are the primary path for charge to flow in the reverse condition. Resistive loss will negatively impact current across the whole voltage range, but typically without strongly impacting fill factor, as the internal bias will not be as strongly affected by the exterior resistance as the $\mathrm{J}_{\mathrm{sc}}$.

As previously mentioned, the conductivity of $\mathrm{P} 3 \mathrm{HT}$ stems from the carboncarbon double bonds in its backbone. In addition, when the polymer chain is ordered in such a way that it forms well aligned rows, the polymer is said to be in an extended conformation, see Figure 12. This is relevant, because the extended ordering of the polymer creates a multiplicity of states in the material's band structure. ${ }^{33}$ The degree to which the polymer is in the extended conformation is sometimes referred to as the conjugation length, and the relationship between this conjugation length and the band properties are well established. There are two major components that are of interest with regards to conformation. Charge conduction within the polymer chain is an order of magnitude faster along the backbone than it is between the chains. Therefore, a polymer layer with a greater conjugation length should be able to transmit charges from one location to another more quickly because the carriers will require fewer interchain "hops" to reach their destination. The second effect of the conformation extension is more subtle, and owes to the development of 
multiple, electrically connected electron states within the molecular orbital of the polymer. Because the optical properties, such as absorption and fluorescence, are set by the interaction between the molecular orbitals, expanding the HOMO and LUMO levels of the polymer through chain extensions should impact such properties. In fact, extending the polymer conformation causes bands to expand, and as they do the effective energy difference between the HOMO and LUMO states decrease. This increases the characteristic wavelengths associated with polymer fluorescence and absorbance, because longer wavelengths are lower energy, in a conversion called a red shift.
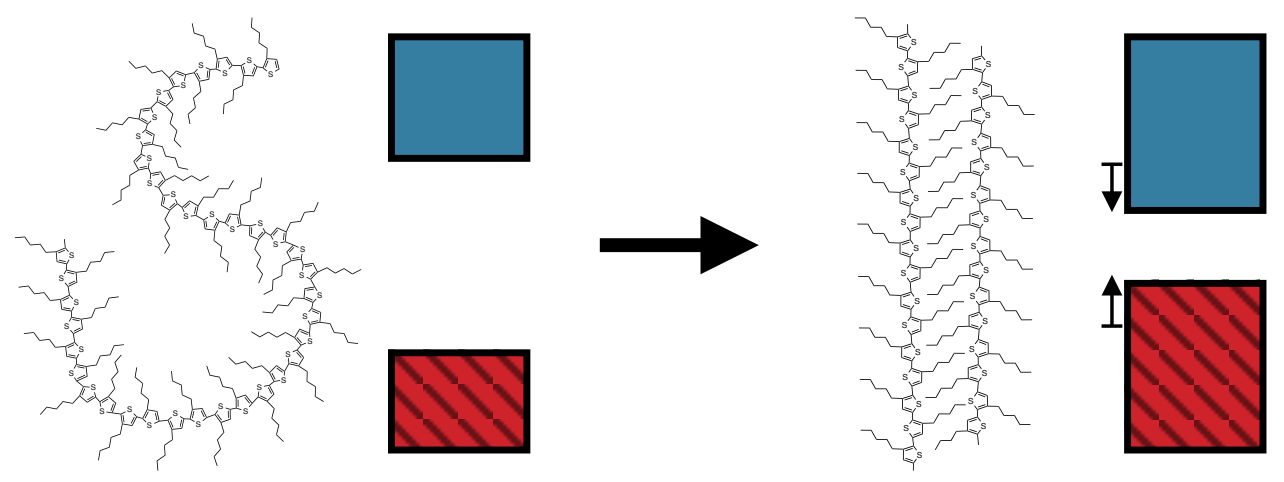

\section{Figure 12 - Effect of polymer conformation on molecular band energies}

\section{HYBRID PHOTOVOLTAICS}

Previous research has shown that the incorporation of metal oxide components into polymer based electronic devices significantly improves device lifetime. ${ }^{14}$ The incorporation of such materials can occur in a number of different ways. One is to incorporate a continuous layer of the metal oxide, in the form of an optical spacer or transparent electrode. While this method is viable, creating continuous metal oxide layers typically requires high temperature processing 
steps which are expensive and do not lend themselves to upscaling. Additionally, metal oxide layers can fatigue under mechanical stresses, causing the layer to fail prematurely in flexible devices. A form of metal oxide layer that does not require thermal processing and is not susceptible to mechanical fatigue is incorporated nanodispersions. The included nanoparticles can still protect the polymer from oxidation by absorbing UV light inhibiting reactions with water and oxygen. ${ }^{34}$ Additionally, careful selection of the metal oxide components could allow them to take a more active role in impacting device performance. Zinc oxide in particular has energy bands comparable with PCBM, making it electrically viable as an acceptor material in place of PCBM. In this case, the device would be referred to as a hybrid, owing its name to its possession of organic and inorganic components in the active layer, shown in Figure 13. Zinc oxide can also be synthesized in nanostructures, such rods and wires, which can be utilized to control the morphology of the polymer layer if there is a favorable interaction between the polymer and nanoparticle phases. Finally, the zinc oxide's conductivity is drastically higher than PCBM, which could allow for the creation of devices with less charge extraction losses and thus higher power conversion efficiencies. ${ }^{35,}, 36$ 


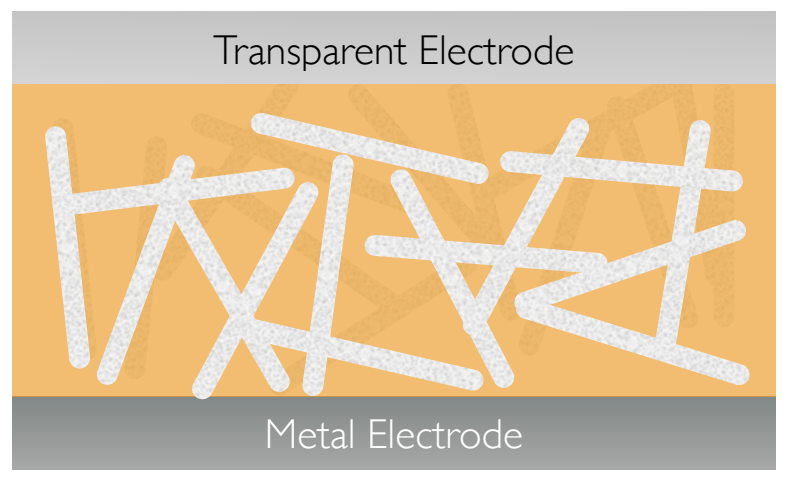

Figure 13 - The structure of a hybrid bulk heterojunction device

The difficulty in creating a hybrid device like the one described above is that $\mathrm{P} 3 \mathrm{HT}$, the most common polymer for photovoltaic applications, doesn't interact favorable with $\mathrm{ZnO} .37$ The alkyl side chains that are added to the polymer backbone to improve the solubility of the P3HT in organic solvents make it far more energetically favorable for the polymer to react with itself, than with the surface of the $\mathrm{ZnO}$. Figure 14 shows a transmission electron microscopy (TEM) image of an applied solution made from pristine $\mathrm{ZnO}$ nanowires and $\mathrm{P} 3 \mathrm{HT}$. The lack of interaction between the polymer and $\mathrm{ZnO}$ phases does not bode well for device performance using these materials, as the efficient separation of excitons created in the polymer phase relies upon electrical connection between the the $\mathrm{ZnO}$ and the P3HT. 


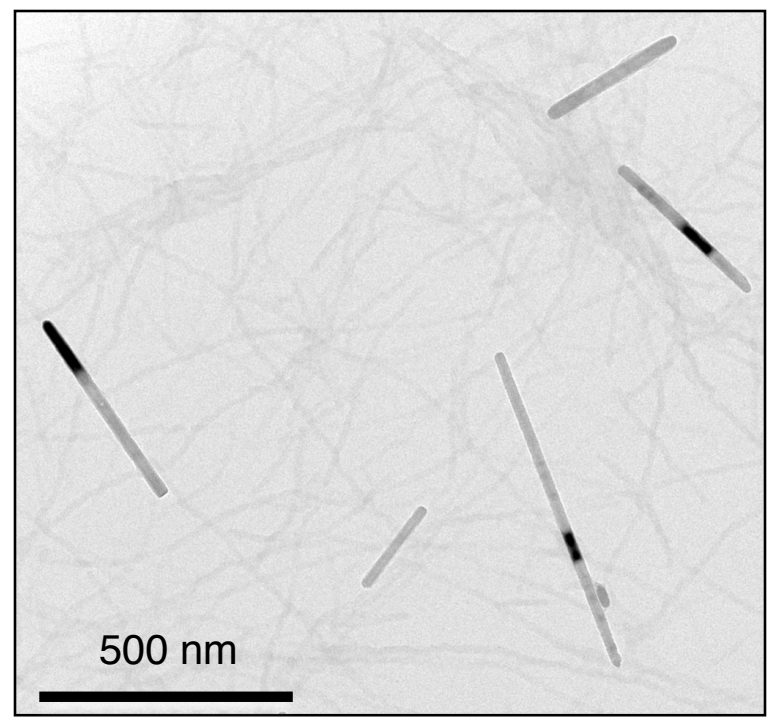

Figure 14 - TEM image of P3HT and pristine ZnO nanowires, displaying no interaction

To make the hybrid structure viable the material phases must be compelled to interact with each other. While there are many ways to accomplish this, there are two methods that are particularly interesting and have the potential for high device performance. The first is $\mathrm{ZnO}$ surface functionalization, in which the nanostructured $\mathrm{ZnO}$ is coated with organic molecules that change its surface properties. The second is the creation of core-shell nanocomposites with the polymer and $\mathrm{ZnO}$ interacting via direct chemical attachment. Both of these methods can solve the issue of donor-acceptor interaction, and they have the added advantage of increasing the processability of $\mathrm{ZnO}$. While it is possible to make dispersions of $\mathrm{ZnO}$ nanowires with $\mathrm{P} 3 \mathrm{HT}$ in organic solvents, the solutions are not stable and the nanowires will rapidly crash out if they are not agitated. The dry $\mathrm{ZnO}$ nanowires in particular are challenging to disperse. Molecules that are used to surface functionalize $\mathrm{ZnO}$ will improve its interactions with both $\mathrm{P} 3 \mathrm{HT}$ 
and organic solvents, which should make it easier to process. Previous research has shown that thiols, carboxylic acids, phosphonic acids, silanes, and siloxanes can successfully react with the surface of $\mathrm{ZnO}$ to form a chemical bond. ${ }^{38-44}$ The two materials selected for $\mathrm{ZnO}$ nanowire functionalization are dodecanethiol and mono-substituted-carboxylic acid tetraphenylporphyrin, shown in Figure 15. The thiol is cheap and easy to apply, while the tetraphenylporphyrin is more difficult to synthesize, but the highly conjugated structure of the molecule could lead to a more conductive functional layer on the nanowire.

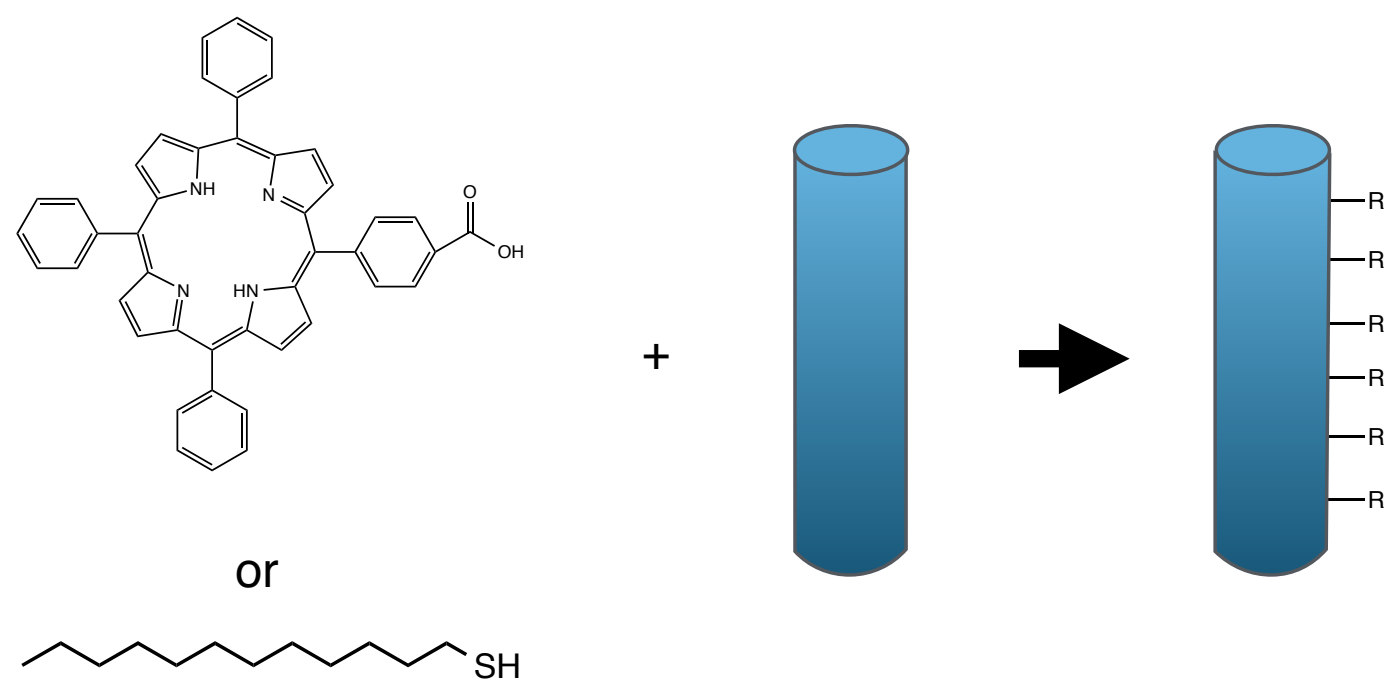

Figure 15 - TPP and DDT functionalization of $\mathrm{ZnO}$ nanowires to improve interactions with solvent and P3HT

Nanocomposite synthesis provides a way to force the interaction between the polymer and the $\mathrm{ZnO}$ phases directly. Nanocomposites can ultimately be formed in two ways; polymer with functionalized end groups can be used to form end on attachment, while polymer with functional groups on the side chain forms 
side on attachment. The general advantage of end on attachment is that the polymer backbone is directly linked to the $\mathrm{ZnO}$ oxide, creating contact points that have very low impedance. By contrast, side on attachment creates more electrical resistance between the polymer and nanowire phases, because the side chains are not conductive, but the contact with the $\mathrm{ZnO}$ should coat the entirety of the surface, decreasing the chances that an exciton will be created too far from a favorable interface. The total coverage of polymer over the $\mathrm{ZnO}$ surface is referred to as a core-shell structure, which may also create highly ordered regions of polymer at the surface of the nanowire. This ordering could be advantageous for charge extraction after exciton dissociation, referring here to the increase in charge mobility along ordered polymer backbones. In this research the side on attachment for the nanocomposite was pursued, with the intention of creating a highly ordered polymer layer for better charge conduction, shown in Figure 16. The nanocomposite was synthesized using $\mathrm{ZnO}$ nanowires and four carboxylated polythiophenes; Poly(3-carboxy-hexyl-thiophene) [P3CHexT], Poly(3-carboxy-pentyl-thiophene) [P3CPenT], Poly(3-carboxy-butylthiophene) [P3CButT], and Poly(3-carboxy-propyl-thiophene) [P3CPropT], the structures of which are all in Figure 17. The molecules are structurally similar to P3HT, but with a carboxylic acid terminated side chain, which will react with $\mathrm{ZnO}$ to form the composite. 

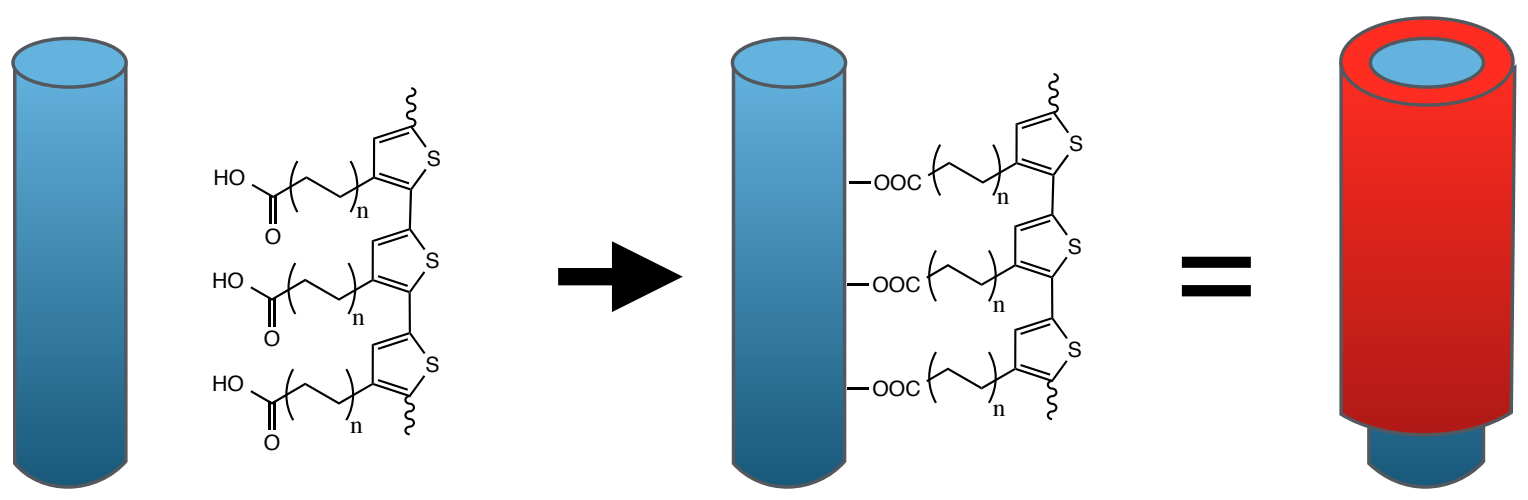

Figure 16 - Scheme for functionalization of $\mathrm{ZnO}$ nanowires via carboxylated polythiophenes. The polymer spontaneously reacts with the nanowire in solution, forming a side on attachment. After sufficient reaction time the $\mathrm{ZnO}$ is completely coated and forms a core-shell structure

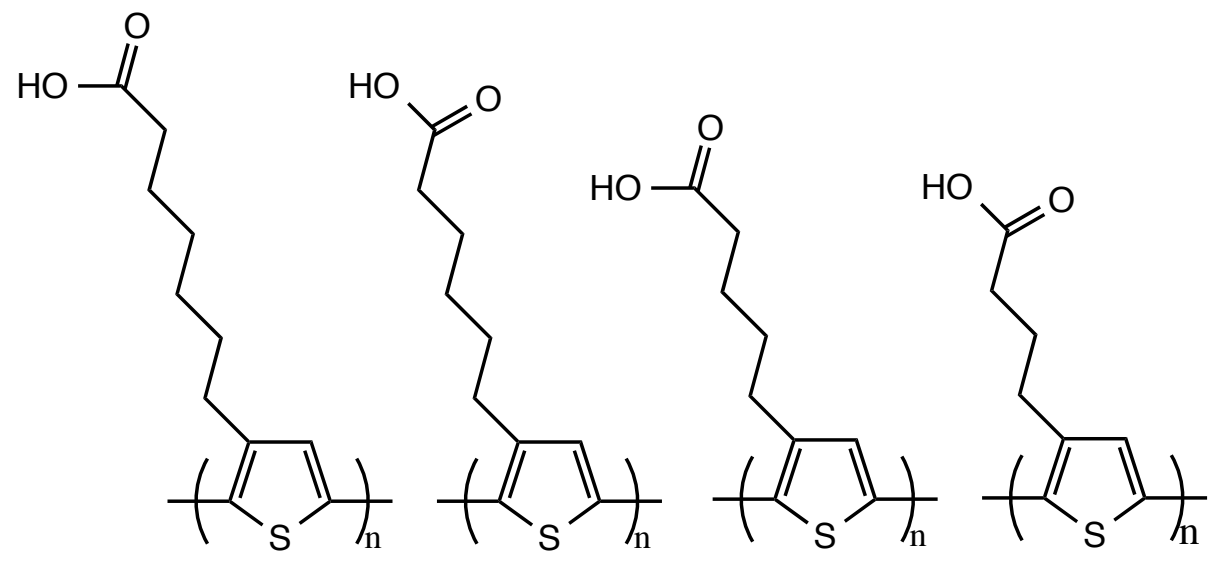

Figure 17 - From left Poly(3-carboxy-hexyl-thiophene), Poly(3-carboxypentyl-thiophene), Poly(3-carboxy-butyl-thiophene), and Poly(3-carboxypropyl-thiophene). 


\section{METHODS AND MATERIALS}

\section{PREPARATION OF ZINC OXIDE NANOWIRES}

To prepare the $\mathrm{ZnO}$ nanowires, zinc nitrate hexahydrate $\left(\mathrm{Zn}\left(\mathrm{NO}_{3}\right)_{2}{ }^{*} 6 \mathrm{H}_{2} \mathrm{O}\right)$ was used as received from Fisher Scientific; with ethanol (EtOH), sodium hydroxide $(\mathrm{NaOH})$ and ethylene diamine obtained from Sigma Aldritch. The zinc nitrate used was in the form of small transparent granules, the sodium hydroxide were in white pellets, and the ethanol and ethylene diamine were transparent liquids. Nanowires were synthesized in batches, with a typical batch of $5.97 \mathrm{mmol}(1.78 \mathrm{~g})$ of zinc nitrate dissolved in $300 \mathrm{~mL}$ ethanol via vigorous stirring at room temperature. After the dissolution of the zinc nitrate, $180 \mathrm{mmol}(7.20 \mathrm{~g})$ of sodium hydroxide were added to the solution and allowed to dissolved under stirring at room temperature. Once the materials were completely dissolved the solution was sonicated using a Heat Systems Misonix Sonicator Ultrasonic Processor XL 2000 with a microtip attachment at 4.8 power for 60 minutes. Then $30 \mathrm{~mL}$ of ethylene diamine was added to the solution and the resulting mixture was sonicated in a bath sonicator for 30 minutes at $50^{\circ} \mathrm{C}$. The resulting solution was then placed in pressurized reaction autoclaves and baked in an oven for 24 hours at $130^{\circ} \mathrm{C}$ (for long rod synthesis) or 4 hours at $100^{\circ} \mathrm{C}$ (for short rod synthesis). The autoclaves were then removed from the oven and allowed to cool on the lab bench before being opened. The final solutions were washed 3 times in deionized water and once in ethanol before being placed in a $50^{\circ} \mathrm{C}$ oven to dry overnight. The resulting white powder was crushed using a mortar and pestle and stored. 
The surface functionalization process for $\mathrm{ZnO}$ nanowires were conducted using $\mathrm{ZnO}$ synthesized as detailed above, chloroform and dodecanethiol obtained from Sigma Aldritch, and mono-substituted-carboxylic acid tetraphenylporphorin obtained from Dr. Chad Immoos. The dodecanethiol and chloroform used were clear liquids, and the tetraphenylporphorin was a purplebrown dusty solid. Functionalization was conducted in batches, with a typical batch of $50 \mathrm{mg}$ of $\mathrm{ZnO}$ nanowires, to which were added $528 \mu \mathrm{mol}(120 \mu \mathrm{L})$ dodecanethiol or $10.2 \mu \mathrm{mol}(6.25 \mathrm{mg})$ tetraphenylporphorin. $\sim 10 \mathrm{~mL}$ of chloroform were then added and the materials were dispersed using a bath solicitor. Once completely dispersed the solution was placed on a vortexer at 1000rpm and allowed to react for $2 \mathrm{hrs}$. The resulting solution was then removed from the vortexer and washed 3 times in chloroform and once in ethanol before being placed in a $50^{\circ} \mathrm{C}$ oven to dry. The dry materials were crushed using a mortar and pestle and stored.

Side on attachment of carboxylated polythiophenes to $\mathrm{ZnO}$ nanowires were conducted using $\mathrm{ZnO}$ fabricated in the process above; Poly(3-carboxyhexyl-thiophene), Poly(3-carboxy-pentyl-thiophene), Poly(3-carboxy-butylthiophene), and Poly(3-carboxy-propyl-thiophene) were used as obtained from Rieke metals; dimethylsulfoxide (DMSO) and pyridine were obtained from Sigma Aldritch and used as delivered. The polymer materials used were a dark clumped fibrous powder, while DMSO and pyridine were transparent liquids. In typical nanocomposite synthesis, $4 \mathrm{mg}$ of carboxylated polythiophene were dissolved in $2 \mathrm{~mL}$ of pyridine or DMSO, then $16 \mathrm{mg}$ of $\mathrm{ZnO}$ nanowires were added 
and the solution was sonicated until completely dispersed. The solution was placed on a vortexer at $1000 \mathrm{rpm}$ and allowed to react. Typical pyridine solutions would reach acceptable functionalization in 1-3 weeks, while DMSO solutions would take more than a month. The progress of the functionalization was tested by removing the solutions from the vortexer and allowing them to settle out on the bench top. A solution that was sufficiently reacted would have minimal orange coloration (polymer) remaining in solution after the composite settled. The solution was then washed with DMSO or pyridine until there was no remaining coloration in the liquid phase (3-5 wash cycles). Samples were then stored in solution to prevent agglomeration, and could be washed into another solvent for testing, or washed into ethanol and left to dry at room temperature to make powdered composite.

CHARACTERIZATION AND ANALYSIS

FOURIER TRANSFORM INFRARED SPECTROSCOPY

Fourier transform infrared spectroscopy (FTIR) was used to evaluate the change in functional groups of the composite as compared to pristine polymer. Tests were conducted using a Nicolet 380 FT-IR spectrometer in attentuated total reflectance mode. The instrument was set to a resolution of $8 \mathrm{~cm}^{-1}$ with a scan accumulation of 1000. All testing was conducted on dried powders for pristine carboxylated polythiophenes and nanocomposites.

THERMAL GRAVIMETRIC ANALYSIS

Thermal gravimetric analysis (TGA) was used to determine polymer loading in composite samples, and to evaluate thermal stability of composites as 
compared to pristine polymer. TGA data was gathered on a TA instruments Q-500. Samples were prepared dry in aluminum pans with sample masses $2-5 \mathrm{mg}$. All samples were held isothermal at $200^{\circ} \mathrm{C}$ for 10 minutes to remove residual solvents, after which the starting masses were measured and the samples were ramped at $10^{\circ} \mathrm{C} / \mathrm{min}$ to $580^{\circ} \mathrm{C}$ under atmospheric oxygen. The first derivatives of sample mass were plotted to determine temperature dependence of thermal decomposition, and remaining sample mass at $580^{\circ} \mathrm{C}$ was used to determine total loading in composite samples.

UV-VISIBLE ABSORPTION SPECTROSCOPY

UV-Visible absorption spectroscopy (UV-Vis) was used to evaluate polymer conformation in nanocomposites as compared with pristine polymer. Tests were conducted using a Jasco V- 550 spectrophotometer with temperature control attachment. All samples were evaluated at $25^{\circ} \mathrm{C}$, with a spectral bandwidth of $2 \mathrm{~nm}$, run speeds ranging from $20 \mathrm{~nm} / \mathrm{min}$ to $100 \mathrm{~nm} / \mathrm{min}$ based on sample response, from $300 \mathrm{~nm}-700 \mathrm{~nm}$ scan range, at a resolution of $0.5 \mathrm{~nm}$. All samples were evaluated in plastic cuvettes with DMSO as the solvent.

\section{UV-VISIBLE FLUORESCENCE SPECTROSCOPY}

UV-Visible fluorescence spectroscopy was used to measure the degree of electrical interaction between the polymer and $\mathrm{ZnO}$ phases in the nanocomposite. Tests were conducted using a Jasco SP-6500 spectrofluoremeter with temperature control attachment. Fluorescence measurements were taken on the same stock solutions used to prepare the samples for UV-Vis absorbance spectroscopy. All fluorescence measurements 
are evaluated with respect to the loading of polymer within the sample; polymer loading of composites was determined via thermal gravimetric analysis and used to normalize fluorescence responses with respect to polymer concentration. Samples were evaluated at $25^{\circ} \mathrm{C}$, with a spectral bandwidth of $3 \mathrm{~nm}$, a test resolution of $1 \mathrm{~nm}$, excitation wavelength of $450 \mathrm{~nm}$, emission wavelengths from $450 \mathrm{~nm}-700 \mathrm{~nm}$, and a scan rate of $50 \mathrm{~nm} / \mathrm{min}$.

\section{X-RAY DIFFRACTION}

X-Ray Diffraction (XRD) was used to verify the synthesis of wurtzite crystal $\mathrm{ZnO}$, and to evaluate the molecular spacing in pure polymer samples. Data was collected using a Siemens D5000 diffractometer with a $1.54 \AA$ Cu Ka source. $\mathrm{ZnO}$ samples were analyzed in powder form from a plastic powder sample holder, while polymer samples were drop cast from pyridine solutions onto microscope slides and dried on a hot plate at $80^{\circ} \mathrm{C}$.

\section{TRANSMISSION ELECTRON MICROSCOPY}

Transmission electron microscopy was used to verify the core-shell structure of the synthesized nanocomposites. Samples were prepared for TEM in dilute ethanol solutions, which were applied drop wise to semipermable carbon film on 300 mesh copper grids purchased from Electron Microscopy Sciences. The resulting samples were transported to Georgia Institute of Technology where they were evaluated using a Hitachi HF2000 transmission electron microscope. 


\section{DEVICE FABRICATION AND CHARACTERIZATION}

All devices were manufactured in the Cal Poly Polymer Electronics Laboratory. Typical device fabrication began with pre-stenciled ITO glass substrates obtained from Rieke Metals, which were scrubbed down with deionized water and inspected for surface contamination. The substrates were then subjected to sonication, first under acetone, then under isopropyl alcohol, for 3 minutes each, with a nitrogen jet for drying in between. From this point the samples were transported to a dust free area where they were subjected to a UV ozone for no less than 30 minutes. A layer of PEDOT:PSS, used as obtained with a $200 \mu \mathrm{m}$ syringe tip filter, was applied to the substrates via spin coating, after which the outer edges of the devices were wiped with water to prevent PEDOT leakage pathways between the electrodes. The devices were thermally annealed on a hotplate at $140^{\circ} \mathrm{C}$ for 10 minutes, then transported into a nitrogen atmosphere glove box where the remainder of the fabrication steps took place. Active layers were applied via spin coating from solutions that were prepared at $20 \mathrm{mg}$ solute per $\mathrm{ml}$ in chlorobenzene, in solute ratios of 1:9 $\mathrm{ZnO}$ :Polymer and 5:5 ZnO:Polymer by weight. Typical solutions were allowed to dissolve/disperse for a minimum of 24 hours on a stirring hotplate at $500 \mathrm{rpm}$ and $50^{\circ} \mathrm{C}$ prior to application to devices. The active material was applied to substrates via spin coating at $600 \mathrm{rpm}$, after which the outer edges of the devices were wiped with a tetrahydrofuran swap to expose the electrode. To complete the devices a metal cathode was evaporated under high vacuum conditions. Typical devices were 
created with aluminum electrodes $100 \mathrm{~nm}$ thick. Select processing steps are shown in Figure 18.

A

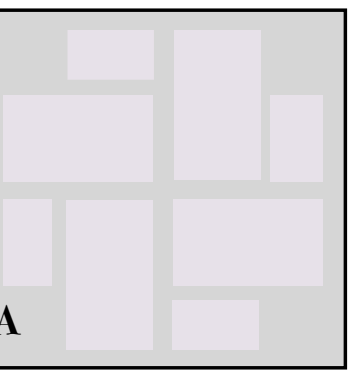

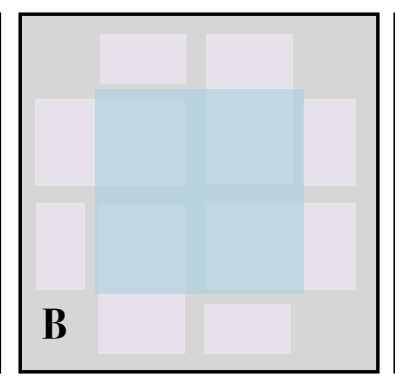
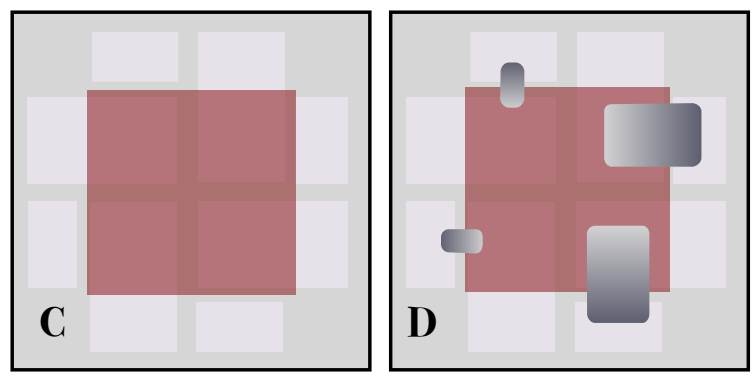

Figure 18 - Device Fabrication steps. A) Begin with prestencilled ITO glass. B) Apply and wipe away PEDOT:PSS. C) Apply and wipe away active layer. D) Evaporate metal electrodes

Differences in solvent compatibility for nanocomposite materials prevented any functional devices from being made using the composite material system.

\section{CURRENT DENSITY-VOLTAGE MEASUREMENT}

Current Density-Voltage Measurements (JV) evaluate cell performance over a wide array of loading by applying a bias and measuring device current. This allows calculation of the maximum power point as well as many other useful pieces of information. Testing was conducted using a Dolan Jenner lamp source directed onto a sample area via optical fibers. Lamp intensity at the test site is estimated to be $18 \mathrm{~W} / \mathrm{m}^{2}$. A Keithly current voltage meter was used to apply the external bias and measure the device current, controlled by an in-house LabView program. Subjected biases for all devices were ranged from $-1 \mathrm{~V}$ to $+1 \mathrm{~V}$ at $0.05 \mathrm{~V}$ increments. Analysis of the resulting JV curves were conducted by an in-house analysis suite that calculated the open circuit voltage, short circuit current, fill factor and power conversion efficiencies of each device. 


\section{OPTICAL DENSITY SPECTROSCOPY}

Solid phase optical density spectroscopy was used for evaluation of active layer absorption in fabricated solar devices. Testing was conducted using an HR 2000+ Ocean Optics spectrometer and an Ocean Optics lamp. Samples were measured using the absorbance mode of SpectraSuite, with a $1 \mathrm{~ms}$ integration time and run averaging of 15 . 


\section{RESULTS AND DISCUSSION}

\section{ZINC OXIDE NANOCOMPOSITE CHARACTERIZATION}

The first step to characterize chemical attachment of carboxylated polythiophenes to $\mathrm{ZnO}$ nanowires was to verify the existence of the chemical bond between the organic and inorganic phases. As mentioned previously, FTIR measures the the response of dipole moments in molecular functional groups, which is uniquely useful in determining the chemical attachment of polythiophenes to the $\mathrm{ZnO}$ surface due to the carboxylic acid group which terminates the side chain. The pristine polymer universally expressed a $\mathrm{C}=\mathrm{O}$ vibrational band at wavenumber $\sim 1700 \mathrm{~cm}^{-1}$ which was unaffected in location by the length of the polymer side chain, shown in Figure 19.47 After reacting with the $\mathrm{ZnO}$ in pyridine, the polymer did not express the same $1700 \mathrm{~cm}^{-1}$ peak, showing instead a diminished response which is likely attributed to the small amounts of polymer that are physically adsorbed onto the already functionalized surface. In the composite, two new spectral peaks ranging from $1400-1470 \mathrm{~cm}^{-1}$ and $1550-1620 \mathrm{~cm}^{-1}$ appear, corresponding to the symmetric and asymmetric bonding that forms between the $\mathrm{COO}$ - and the $\mathrm{Zn}^{2+}$ surface. The suppression of the $\mathrm{C}=\mathrm{O}$ vibrational mode and the appearance of the two COO- vibration peaks gives good evidence to support a chemical linkage between the carboxylated polythiophenes and the $\mathrm{ZnO}$ nanowires. 


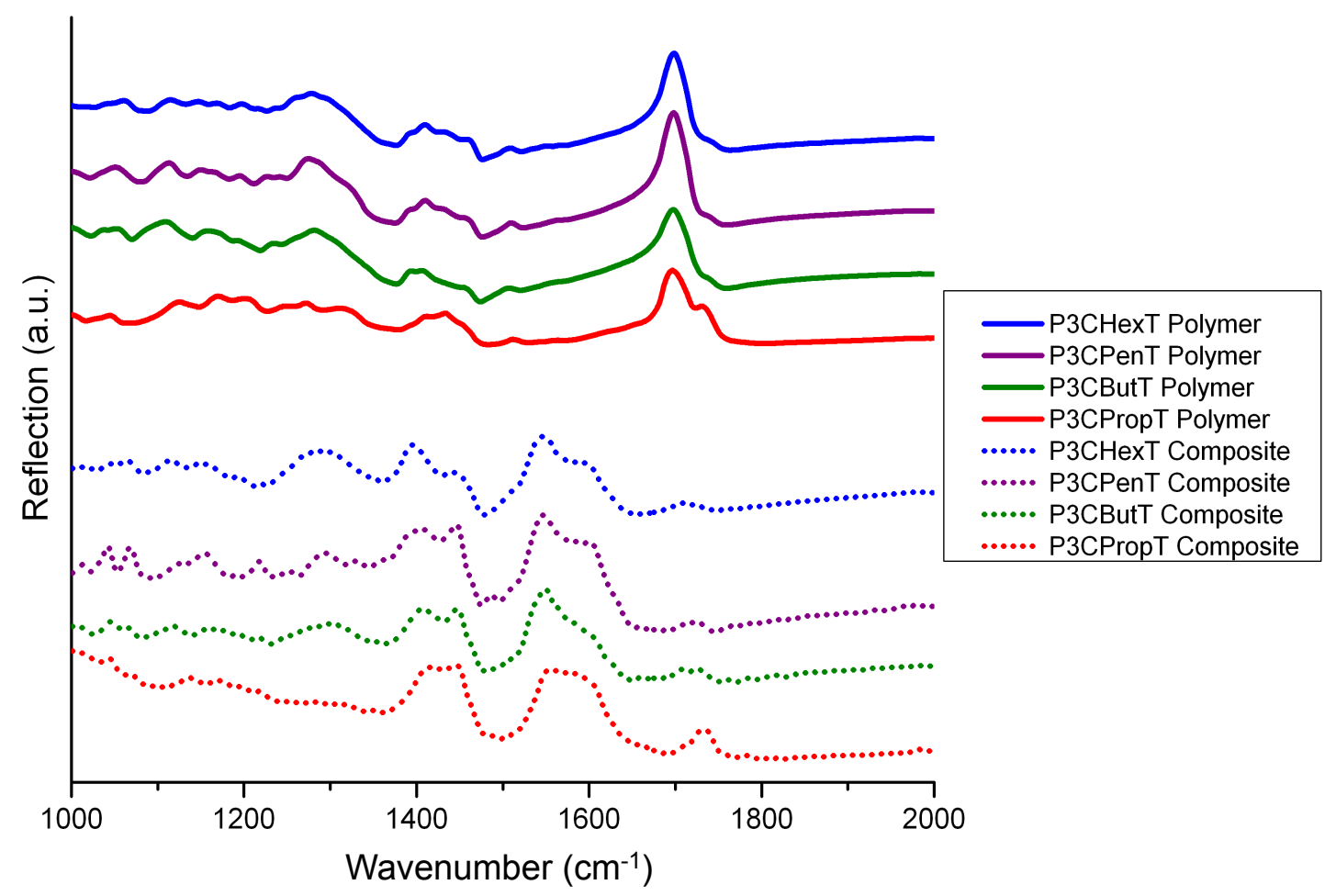

Figure 19 - FTIR spectra of carboxylated polythiophene and ZnO polythiophene nanocomposites synthesized in pyridine.

With the nature of the bonding established, it was necessary to measure the polymer loading in the composite before proceeding forward with further testing. Thermal gravimetric analysis was used to find the polymer loading within the composite via total thermal decomposition, and also was used to investigate the thermal stability of the polymer as compared to the composite. The loadings for the composites were 8.5\% P3CPropT in composite, 10.0\% P3CButT in composite, 14.7\% P3CPenT in composite, and 10.3\% P3CHexT in composite, all shown in Figure 20. The levels of these loading were determined primarily by the amount of polymer in the reaction solution; this research does not imply that 
P3CPenT forms significantly higher loading composites that the other carboxylated polymers used. Previously made composites have had loadings as high as $45 \%$ polymer, but difficulties redispersing those materials has made lower loading composites more desirable.

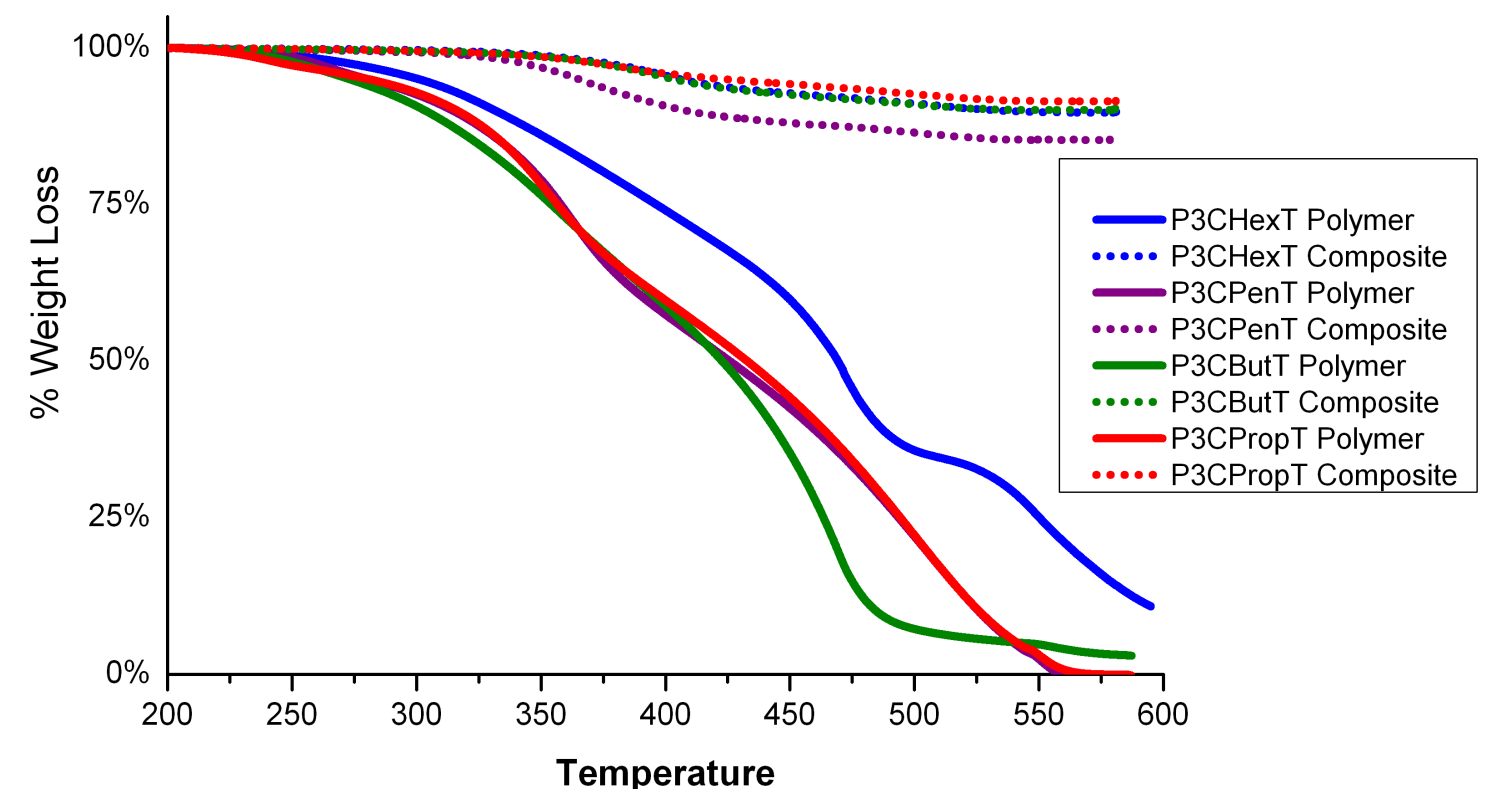

Figure 20 - Thermal gravimetric analysis of P3C(n)T nanocomposites

Thermal stability of the polymer is related largely to the strength of the chemical bonds within the molecule. The derivative of mass loss with respect to temperature from the TGA shows that the first (lowest temperature) thermal decomposition peaks in the composite occur at slightly higher temperatures than for the polymer alone, see Figure 21. From this it is concluded that attachment of the polymer to the zinc oxide makes for a more thermally stable whole. This could translate into a more robust material overall. 


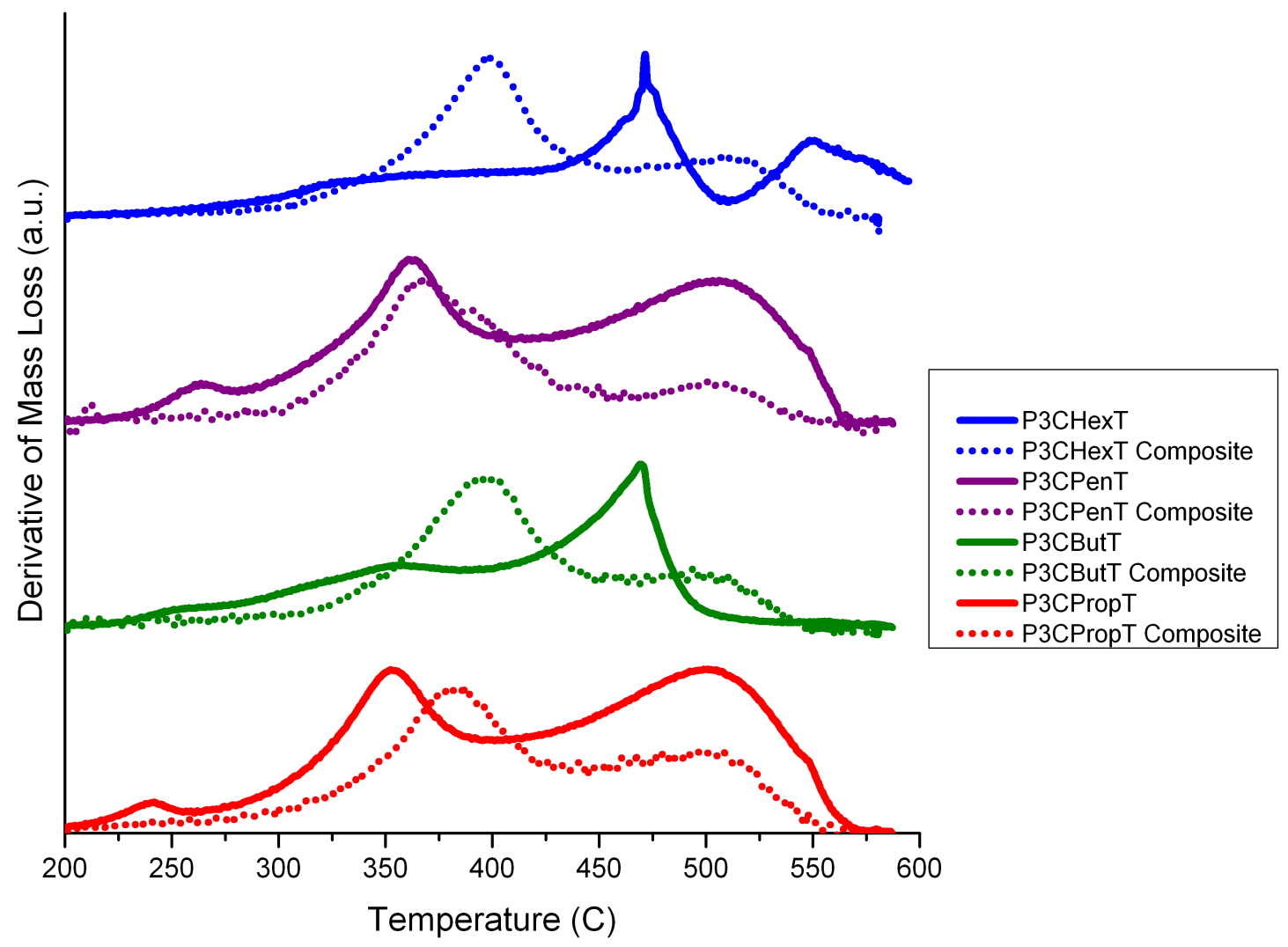

Figure 21 - TGA derivatives comparing composite decompositions with polymer decompositions

After confirming chemical linkages between the polymer and nanowire phases and the amount of polymer contained in each composite, it was important to determine any sort of organization to the attachment of the material. TEM imaging, shown in Figure 22, has the resolution and the contrast to distinguish the nanowire from its polymer shell, so scanning the edge of the wires effectively gives a cross sectional image of the composite sample. At low magnifications it is immediately apparent that there is no free polymer, especially when compared with the TEM imaging of pristine $\mathrm{ZnO}$ and $\mathrm{P} 3 \mathrm{HT}$, see Figure 14. At higher magnifications a lightly contrasted polymer layer, ranging from $3 \mathrm{~nm}$ to $30 \mathrm{~nm}$ 
thick, covers the ZnO's exterior. The layer of polymer closest to the $\mathrm{ZnO}$ surface is covalently linked to the nanowire, but the remainder of the polymer chains are held in place by their structural backbone and favorable hydrogen bonding between the remaining carboxylic acid groups. This favorable interaction amongst the polymer repeat units can allow the shells of multiple nanowires to interact, as shown in Figure 22.b. This potential interaction between composite shells bodes well for device performance, as the polymer shell of the composite is not restricted to interacting with its own nanowire. On extremely high magnification, it is apparent that some areas of the nanocomposite are coated with a thin of polymer roughly $3 \mathrm{~nm}$ thick. The existence of these thin regions on the nanowire surface speaks to a polymer chain that is likely in an extended conformation, which means that the polymer has larger portions of the backbone in an uninterrupted line than it would freely.

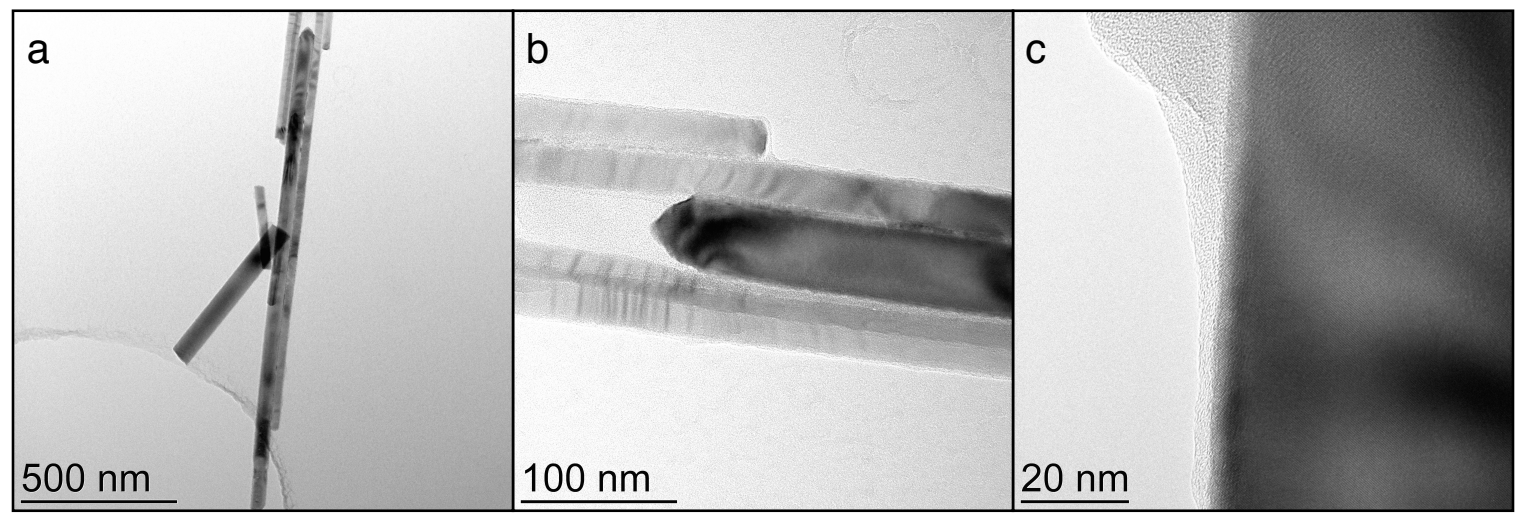

Figure 22 - TEM imaging of ZnO polythiophene nanocomposite. Rods fabricated exhibit a variance in length, but are relatively homogenous in diameter (top left). The polymer shell of the nanocomposite is bonded to the nanowire but can interact with polymer from other, similarly bonded nanowires (top right). The shell of the polymer is believed to be capable of forming monolayers given lower loadings, while the excess polymer can physically adsorb onto the surface of the modified nanowire (bottom left). Excessive polymer loading can lead to clumps of functionalized nanowires that are very difficult to disperse (bottom right). 
Through the process of synthesis and workup of multiple composite groups, a link was determined to exist between the processing of the composite and difficulty in dispersing. Most of all, it was observed that that drying the materials in an oven, which was set to $70^{\circ} \mathrm{C}$, created composites that could not be redispersed in solution, even after hours of sonication. Higher polymer loadings seemed to exacerbate this problem, so TEM imaging of a high loading, oven dried composite material was taken to identify the issue shown in Figure 23. It was found that nanorods formed large clusters under these conditions, which were stable and did not break down under sonication, even in dilute solutions. The polymer binding the clump together contained much more material than could be attributed to surface attachment, so it appears that the physically adsorbed polymer is interacting strongly with both itself and polymer chains that are attached to the surface of the $\mathrm{ZnO}$. The chains are interacting with each other so strongly, in fact, that they overcome the natural tendency of the polymer to interact favorably with the solvents used to dissolve the carboxylated polythiophenes. 


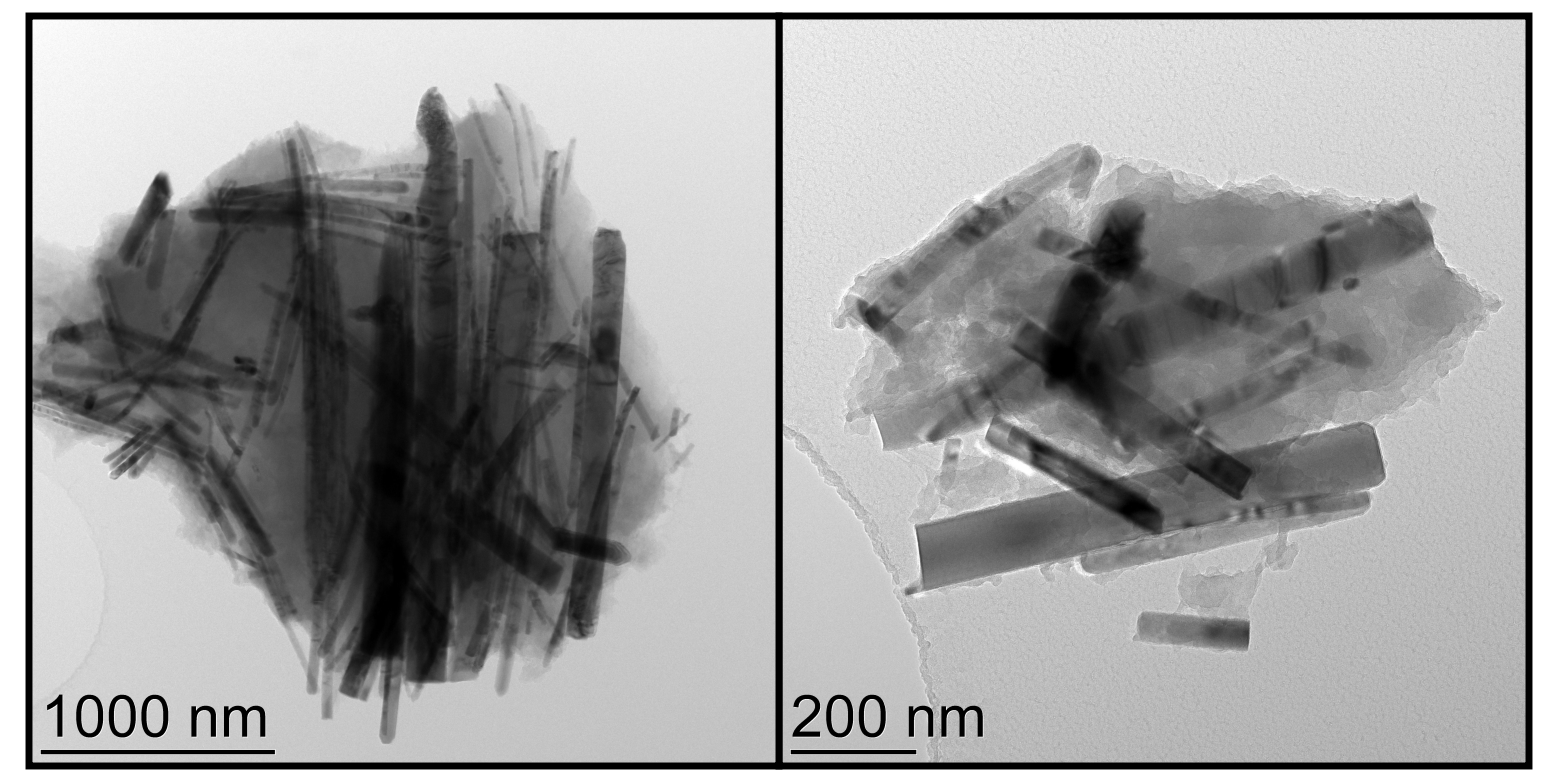

\section{Figure 23 - TEM images of high loading ZnO polythiophene nanocomposites}

While TEM does give the structural element of the core shell composite it is does not yield much information as to the effect of the side-on attachment on polymer alignment, except that very thin layers may imply a more extended conformation. To test the effect of linkage on the polymer UV-visible absorbance spectroscopy was used, shown in Figure 24, in conjunction with the previously mentioned relationship between polymer conformation length and optical properties. UV-Vis spectra were collected for $\mathrm{ZnO}$, pristine polymer samples and nanocomposite samples, all measured in DMSO. In all samples containing $\mathrm{ZnO}$ the characteristic absorption peak at $375 \mathrm{~nm}$ maintained the same location. This is strong evidence that minimal impact is had on the electrical properties of the bulk $\mathrm{ZnO}$ as part of the composite formation process. Pristine polymer samples in DMSO showed peak locations in the absorbance spectra that were dependent on polymer side chain length, from $450 \mathrm{~nm}$ (P3CPropT) to $475 \mathrm{~nm}$ (P3CButT) to 
490nm (P3CPenT) to 550nm (P3CHexT). The increase in wavelength

corresponding to increasing side chain length indicates the polymer conjugation length is increasing with the size of the side chain. This in turn indicates that polymer-polymer interactions are becoming more dominant compared with polymer-solvent interactions as side chain length increases. The P3CPenT and P3CHexT samples also show a secondary absorption peak occurring $~ 600 \mathrm{~nm}$, which has been shown to be a characteristic absorption peak for crystalline $\mathrm{P} 3 \mathrm{HT}$. This further indicates that longer chain side chains are decreasing the solvating power of DMSO for this polymer system.

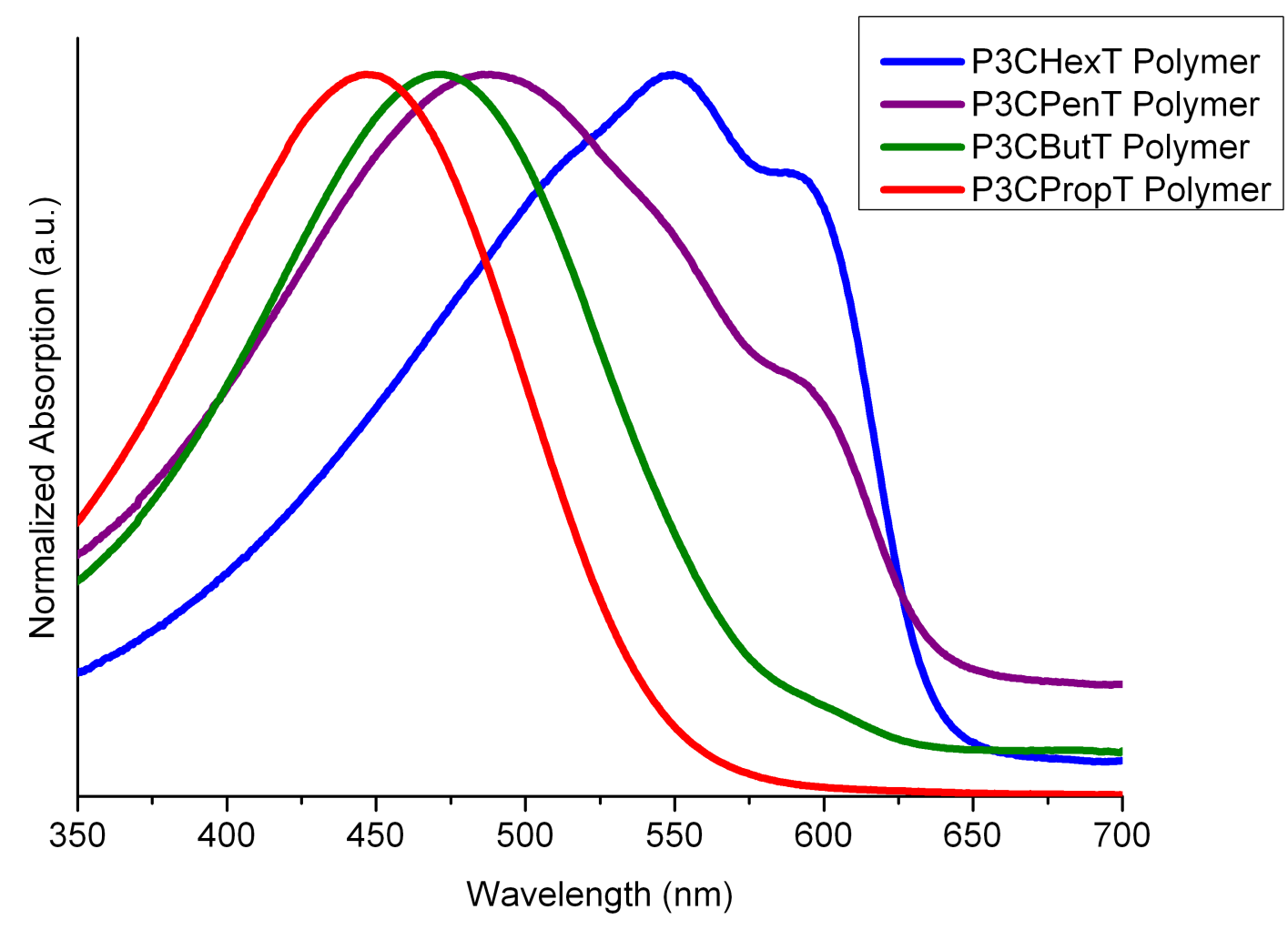

Figure 24 - UV-Vis of carboxylated polythiophenes in DMSO 
$\mathrm{ZnO}$ is not a good absorber of visible light but it does have a strong absorbance peak in the near ultra-violet at about 370nm, as shown in Figure 25. UV-Vis spectroscopy of the $\mathrm{ZnO}$ shows that it has a diminishing absorption, trailing from that $370 \mathrm{~nm}$ peak down to nearly full transmittance by $600 \mathrm{~nm}$, but it is likely that the observed slope is the result of scattering by the $\mathrm{ZnO}$ nanoparticles and not absorption. $\mathrm{ZnO}$ is used as a pigment filler with reasonable hiding power owing to it's high index of refraction, ${ }^{52}$ and the nanoparticles used in this research are on the same order of size as the wavelength of visible light, so the diminished scattering effect at longer wavelengths could be attributed to the longer wavelength light being less likely to encounter the $\mathrm{ZnO}$. This would also explain the increased scattering effect of the composite, as opposed to the pristine $\mathrm{ZnO}$. The clusters of nanowires would mitigate some of the wavelength dependence of the scattering, increasing the observed absorbance of the composite materials at larger wavelengths. 

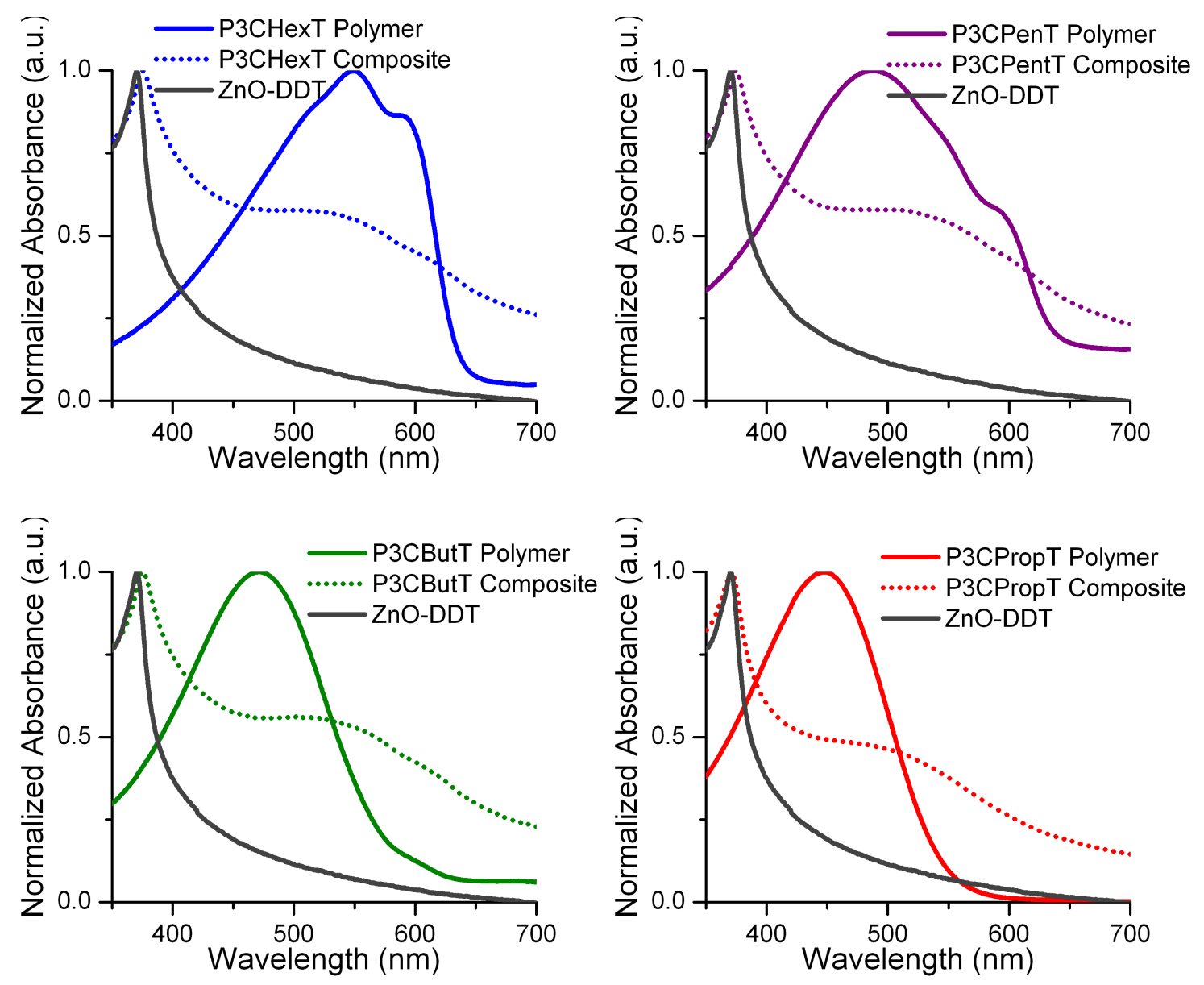

Figure 25 - UV-Vis absorbance spectroscopy of ZnO nanowire polythiophene composites in DMSO

To compare the polymer component of the nanocomposite with the pristine polymer, the $\mathrm{ZnO}$ optical signature is subtracted off. The remaining values are renormalized, and presented in Figure 26. The composite materials show a red shift in the peak value that varies from $450 \mathrm{~nm}$ to $500 \mathrm{~nm}$ (P3CPropT), from $475 \mathrm{~nm}$ to $535 \mathrm{~nm}$ (P3CButT), from $490 \mathrm{~nm}$ to $530 \mathrm{~nm}$ (P3CPenT) and a broadening of the preexisting peak at 550nm (P3CHexT), see Figure 26. As stated before, the positioning of the peaks is impacted by the degree of polymer ordering, where more ordered materials with longer conjugation lengths will have 
optical signatures shifting towards the red. It appears that DMSO is a poor enough solvent for $\mathrm{P} 3 \mathrm{CHexT}$ that the polymer component of the composite is not significantly more extended than the pristine polymer in solution.
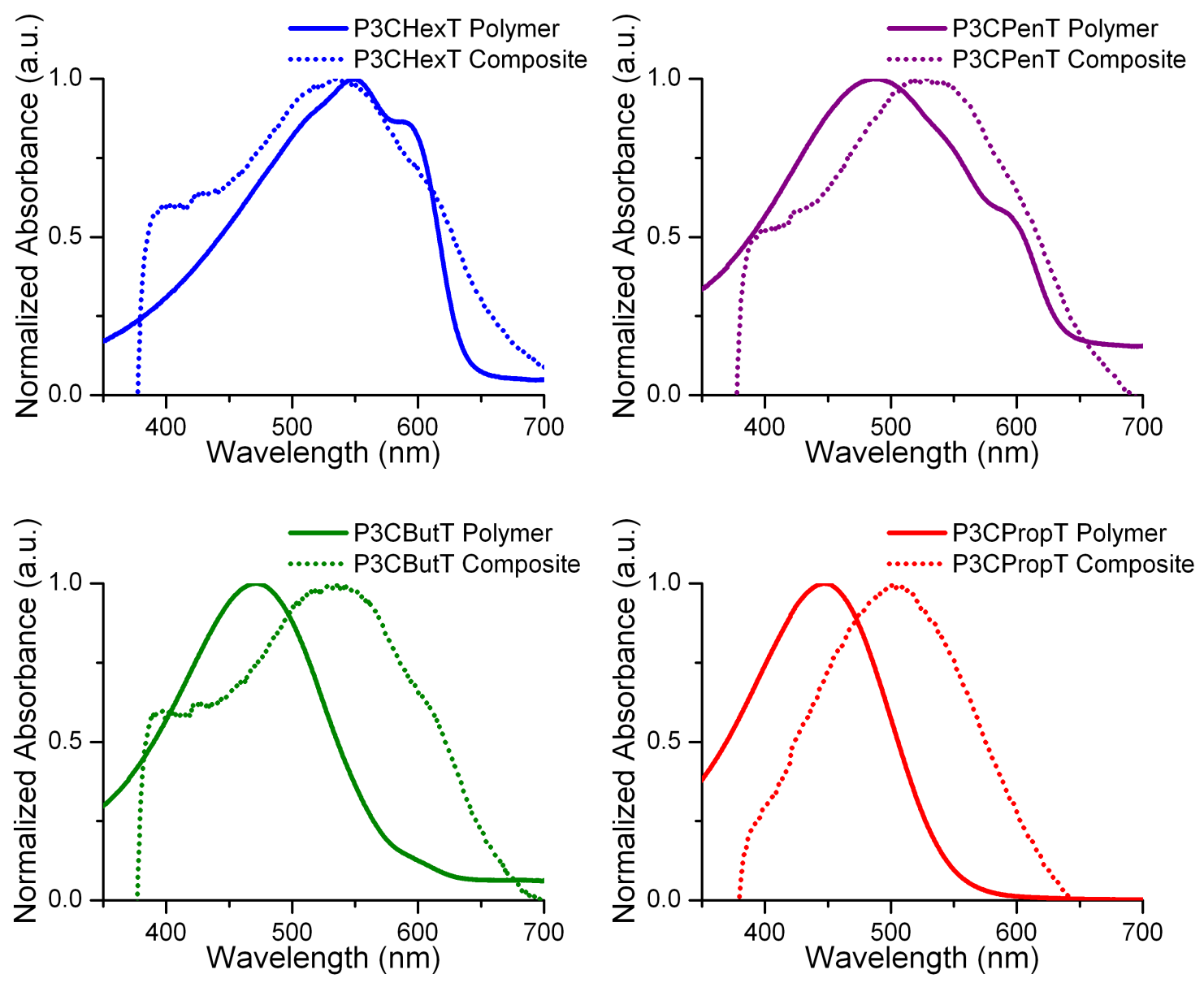

Figure 26 - UV-vis absorbance spectroscopy comparing polymer and composite materials in DMSO

It is worth noting that Figure 27 shows the polymeric component of the nanocomposites for P3CHexT, P3CPenT and P3CButT have similar peak structures in UV-Vis absorbance (530nm), but that the P3CPropT composite 
shows a different composite peak location (500nm). This is likely due to the more favorable interactions between the polymer material and the solvent. TEM imaging showed that P3CPenT nanocomposites had significant polymer material physically adsorbed to the surface. The transition from a 3 to a 4 carbon length side chain may represent a transition from entropically favorable disorder owing to polymer solvent interaction, and energetically favorable order dictated by polymer-polymer interactions.

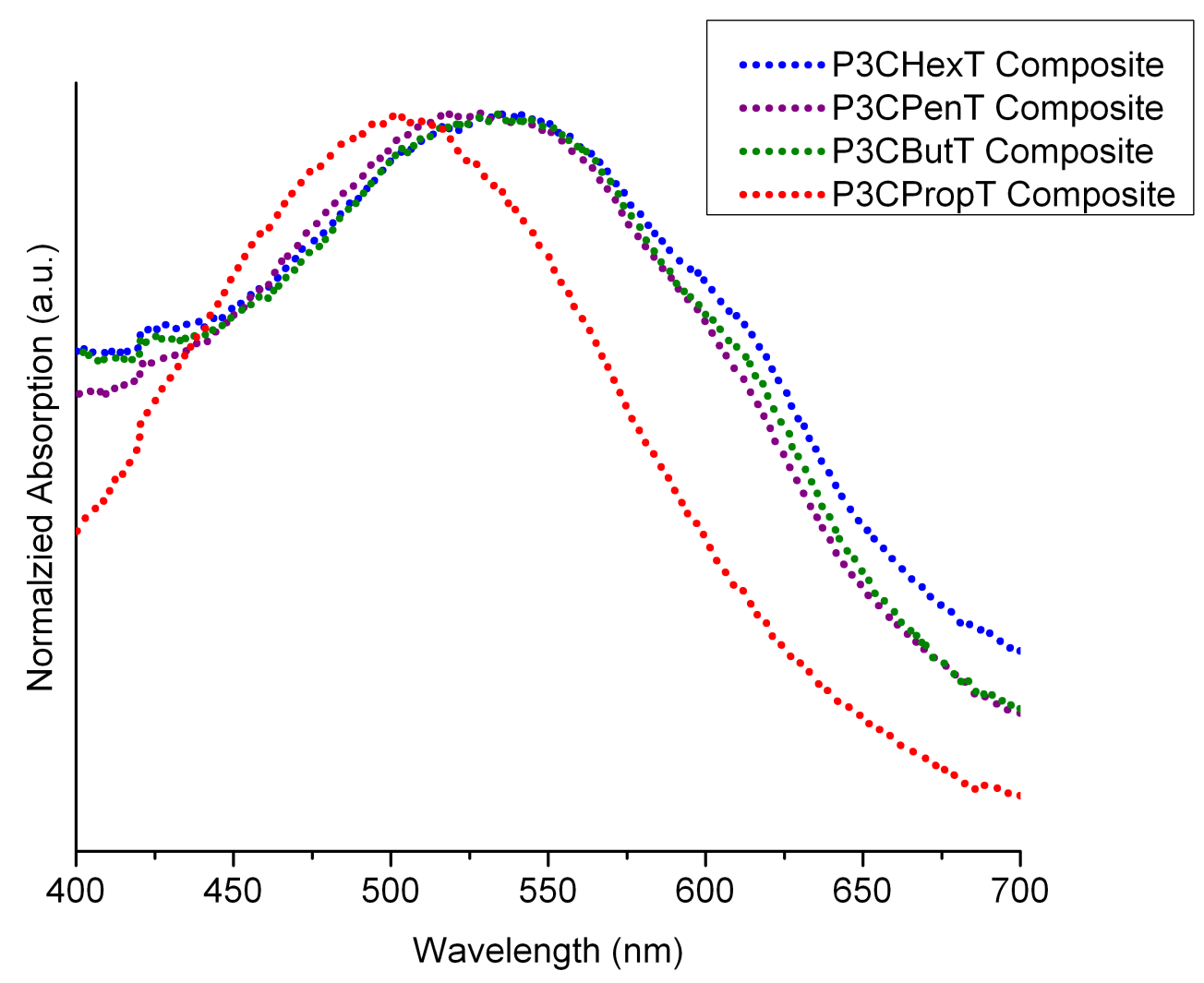

Figure 27 - UV-Vis absorbance spectroscopy of the polythiophene shell of nanocomposite in DMSO 
The most important question with regards to photovoltaic device performance is the nature of the electrical interaction between the polymer and $\mathrm{ZnO}$ phases of the nanocomposite. Good electrical interaction will allow the $\mathrm{ZnO}$ to capture electrons that migrate to the material interface in the form of excitons, while poor interaction will impede them. Fluorescence spectroscopy can evaluate this interaction by providing the polymer component of the nanocomposite with light that is energetic enough to excite electrons from the HOMO to the LUMO state and measuring characteristic fluorescent emissions. If the polymer is electrically connected to a material that can capture electrons from excited states, the reemission of characteristic wavelengths will be significantly reduced, or quenched. Figure 28 shows the fluorescent properties of each polymer and its corresponding nanocomposite normalized to the polymer concentration in the solution. The total quenching of the polymer fluorescence in the composite materials speaks strongly to the favorable electrical interaction between the polymer and $\mathrm{ZnO}$ phases in this nanocomposite. The different intensities of fluorescence between pure polymer samples can be attributed once again to the strength of solvent-polymer interactions as compared to polymerpolymer interactions. Longer side chain units make self interaction more favorable, so the pristine $\mathrm{P} 3 \mathrm{CHexT}$ forms its own ordered regions, even in solution, which makes it possible for the polymer to transport charges more easily and find areas where the excited electrons can de-excite via non radiative transitions. It is also worth noting here that the fluorescent emission of the P3CPropT composite is an order of magnitude greater than that of any of the 
other composite materials. The lack of shift in peak position, as well as this less significant quenching effect seem to corroborate the statement that P3CPropT has sufficiently strong polymer-solvent interaction to maintain mobile polymer chains in DMSO, even when portions of those chains are chemically grafted to the $\mathrm{ZnO}$ surface.

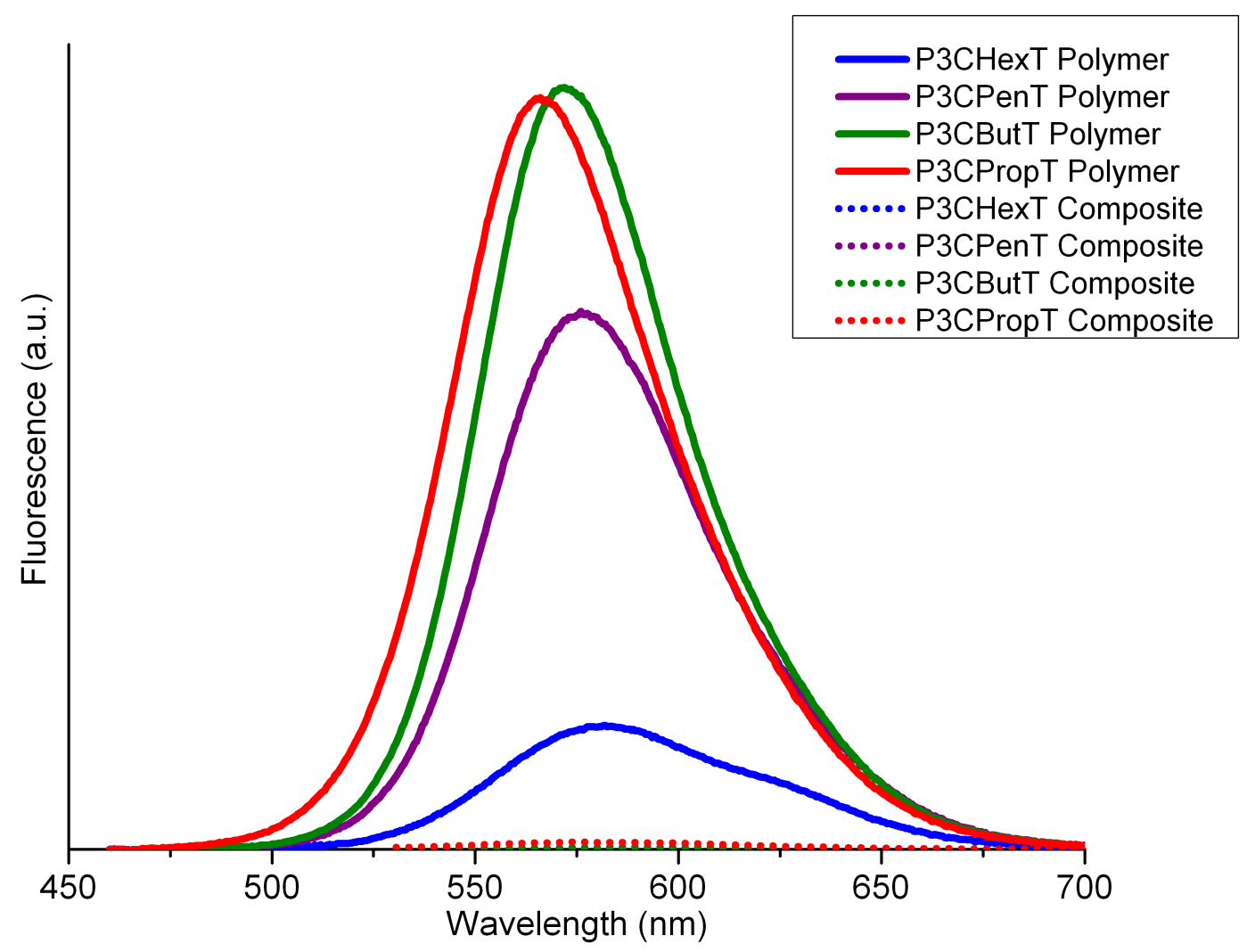

Figure 28 - Fluorescence spectra for carboxylated polythiophene and polymer nanocomposite in DMSO excited at 450nm.

With regards to the pure polymer, the normalized fluorescence spectra still shows signs of the characteristic red shift that is associated with the ordering that accompanies longer chain length structures. The polymer peaks, shown in Figure 29 as 566nm for P3CPropT, 572nm for P3CButT, 576nm for P3CPenT, 
and 582nm for $\mathrm{P} 3 \mathrm{CHexT}$ are cleanly ordered based on side chain length. It is curious that, while the absorbance spectra of P3CHexT and to a lesser extent P3CPenT show a strong secondary absorption peak associated with their crystalline phase, the secondary peak characteristics in the fluorescence spectra are comparatively diminished. Part of this is no doubt associated with the higher conductivity of the crystalline phase, which would make it more likely for charges to reach an area where they can recombine non-radiatively.

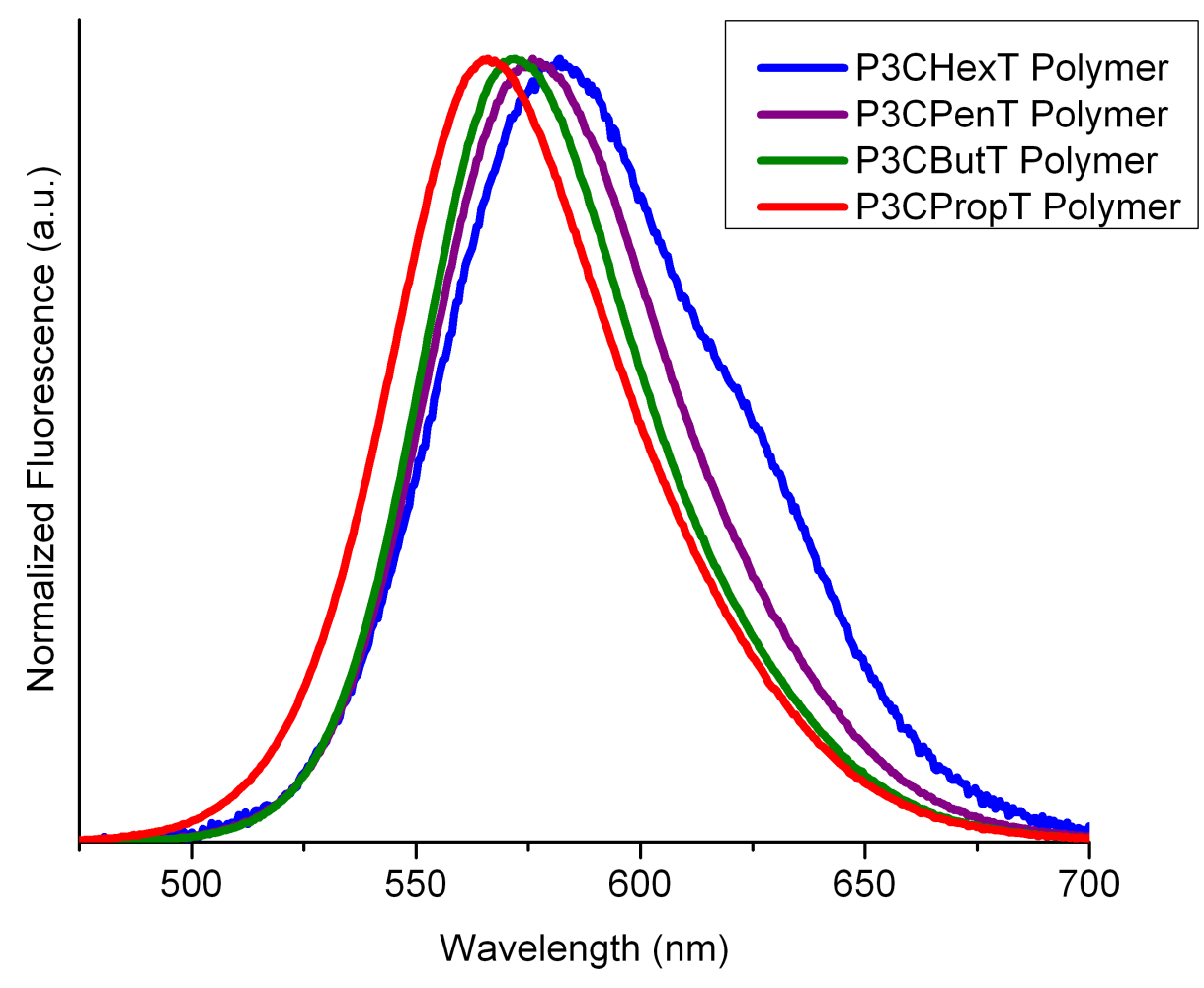

Figure 29 - Fluorescence spectra for carboxylated polythiophene in DMSO, excited at 450nm

For fluorescence as well as absorption, the formation of the nanocomposite causes a red shift in the optical-electronic properties of the 
polymer. We have shown that the composite layer's fluorescences demonstrates a universal red-shift when compared to the polymer alone, shown in Figure 30. While most of the peaks retain the same general shape with a red shift applied, the P3CPropT composite shows a smaller total red shift but a fluorescence broadening that the other samples do not present, see Figure 31. One explanation for this phenomena would be that the P3CPropT composite maintains two distinct phases; there is a portion of the polymer chain that is chemically linked to the surface and therefore unable to move, extending the conformation length, and another portion of the chain that is effectively free to move, thus bearing a diminished conformation length. 

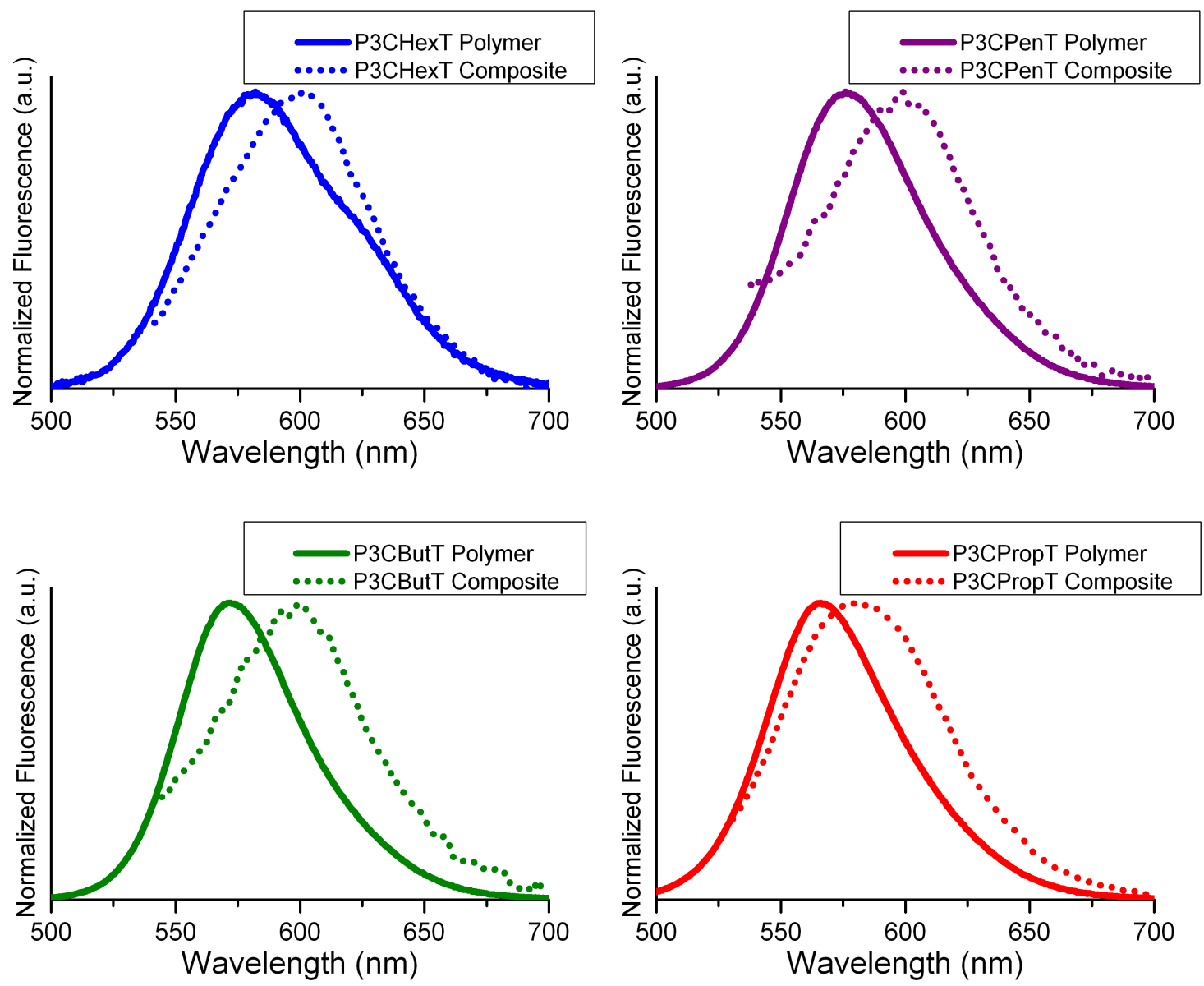

Figure 30 - Fluorescence spectra for carboxylated polythiophenes and nanocomposites with $\mathrm{ZnO}$ nanowires in DMSO, excited at $450 \mathrm{~nm}$ 


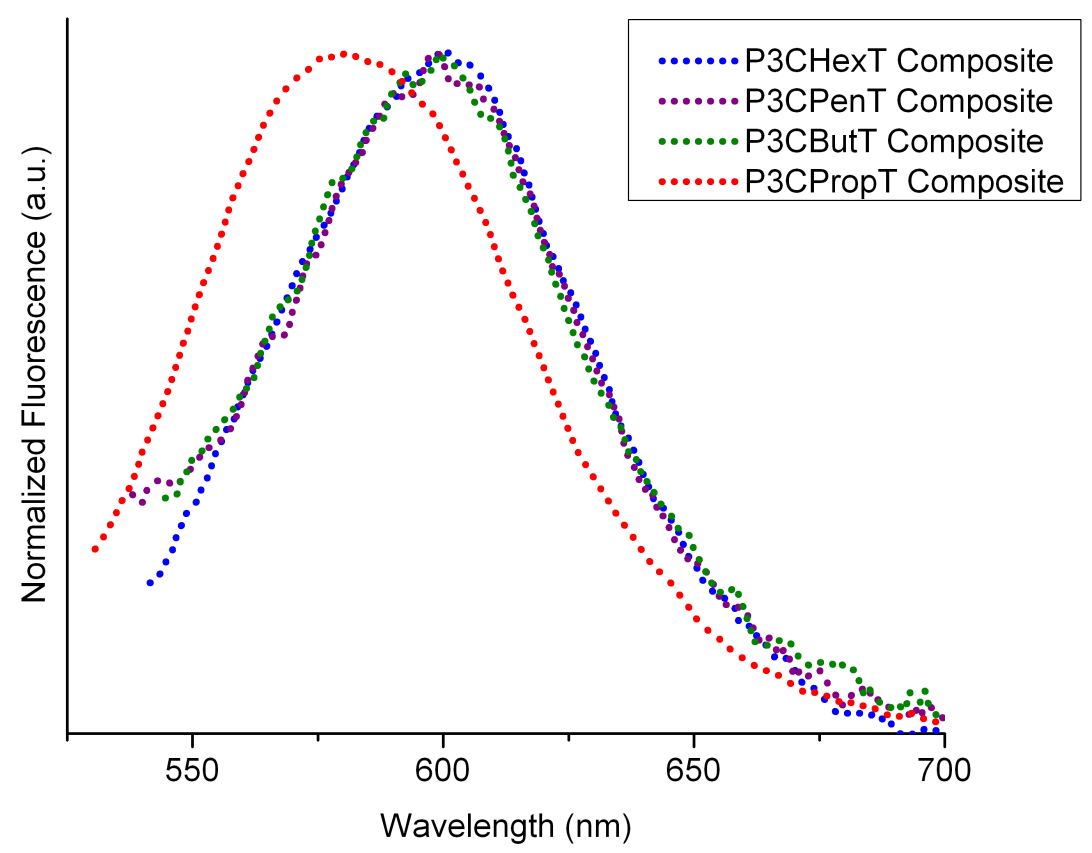

Figure 31 - Fluorescence spectra for polymer composites in DMSO, excited at $450 \mathrm{~nm}$

\section{FUNCTIONALIZED ZNO NANOSTRUCTURES FOR HYBRID SOLAR CELLS}

The functionalized $\mathrm{ZnO}$ nanostructure approach to solving charge extraction issues for $\mathrm{ZnO}$ P3HT hybrid solar cells has a much more straightforward implementation than the polymer nanostructure composite approach. Functionalization of $\mathrm{ZnO}$ via TPP and DDT was confirmed immediately by the solubility of the functionalized nanowires in organic solvents like chloroform and chlorobenzene, and the comparable LUMO level between ZnO and PCBM meant that no modification to electrode structure or device design was needed. The following electrical data was obtained for 12 devices fabricated using the method outlined in methods and materials, herein referred to as 1:9 ZnO-DDT:P3HT, 5:5 ZnO-DDT:P3HT, 1:9 ZnO-TPP:P3HT and 5:5 ZnO- 
TPP where the ratios refer to masses used to create active material solutions. The first piece of collected data, the open circuit voltages shown in Figure 32, seem to indicate that the TPP- $\mathrm{ZnO}$ devices have well performing material interfaces, and that the addition of the TPP functionalization is not significantly changing the electrical characteristics of $\mathrm{ZnO}$. It appears, however, that the surface functionalization of $\mathrm{ZnO}$ with DDT is changing some elements of the electrical interaction between the nanowire and the polymer. The decreased magnitude of the $\mathrm{ZnO}$-DDT device $\mathrm{V}_{\mathrm{oc}}$, and the increased variance in $\mathrm{V}_{\mathrm{oc}}$ indicate that this may be one of two problems. Either the DDT is acting like an insulator and preventing effective electrical interaction between the phases, in which case we should see a significant decrease in forward pass current and $J_{\mathrm{sc}}$, or the surface functionalization is changing the effective energy of the $\mathrm{ZnO}$ at the interface, decreasing the $\mathrm{V}_{\mathrm{oc}}$, and the imperfect coverage of DDT on the $\mathrm{ZnO}$ surface is leading to greater device variance.

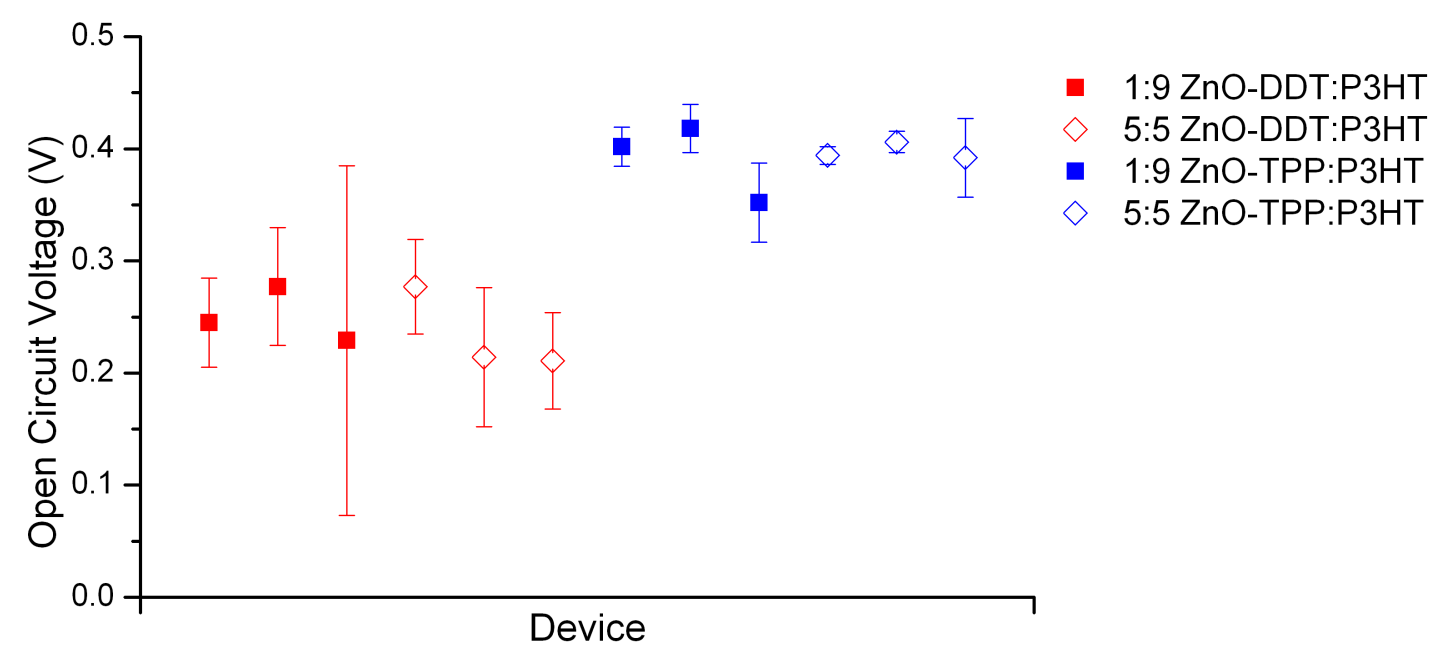

Figure 32 - Open circuit voltage plot of surface functionalized ZnO:P3HT bulk heterojunction hybrid devices 
The short circuit current data, shown in Figure 33, immediately contradicts the insulating shell theory for the DDT-ZnO, as the short circuit currents of the TPP and DDT functionalized nanowires are comparable in magnitude. It is clear from this test that the high polymer loading of 1:9 functionalized $\mathrm{ZnO}: \mathrm{P} 3 \mathrm{HT}$ creates polymer regions that are too large, and does not provide this solar device with sufficient interfaces to extract the generated excitons.

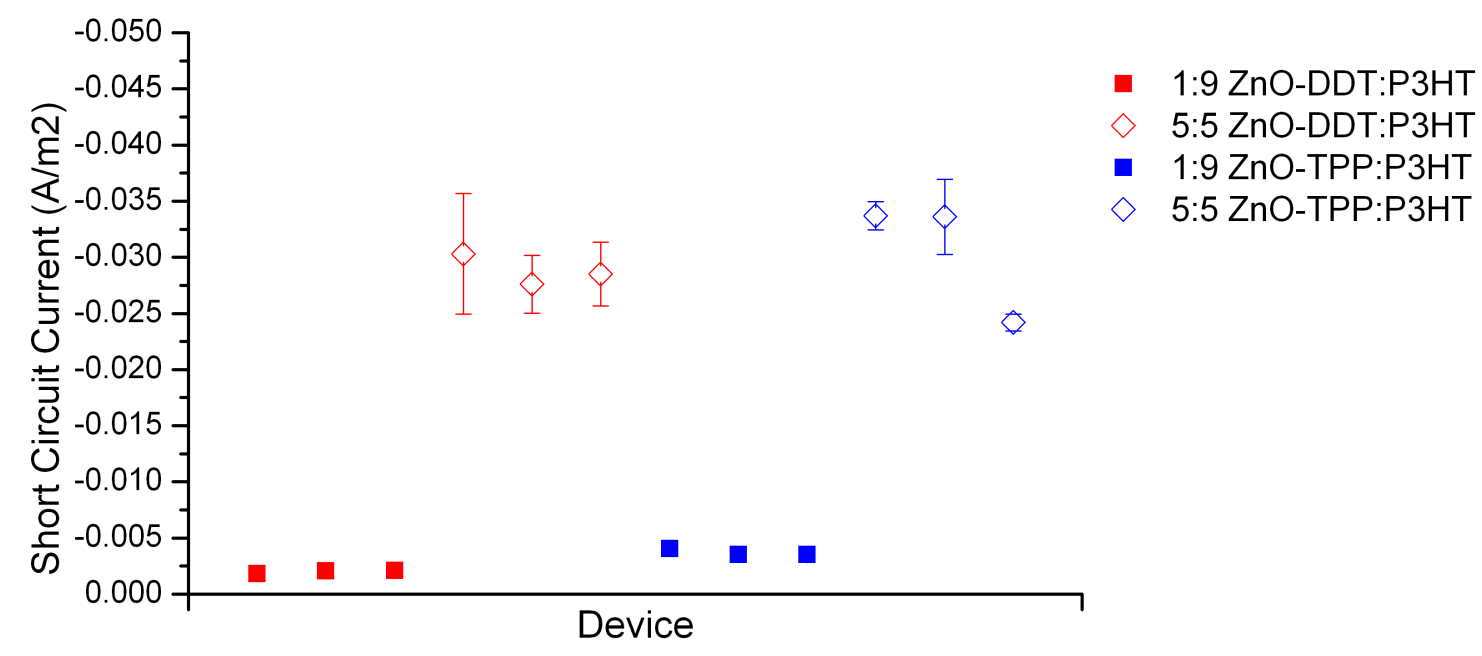

Figure 33 - Short circuit current plot of surface functionalized ZnO:P3HT bulk heterojunction hybrid devices

The next analysis step is to look at characteristic JV curves for the TPPZnO and DDT-ZnO devices. Dark curves in particular, which are the electronic responses of the devices when under no illumination, give insight into the inner working of these materials. The plotted values of the ZnO-TPP and ZnO-DDT dark curves for a 5:5 devices, shown in Figure 34, indicate that the forward pass voltages of both devices are well aligned. In fact, at $+1 \mathrm{~V}$ the forward pass currents are within $5 \%$ of each other. However, in the reverse bias case the TPP-ZnO:P3HT device shows significantly higher current leakage than the DDT- 
$\mathrm{ZnO}: \mathrm{P} 3 \mathrm{HT}$ device. Previous device fabrications with the nanostructured $\mathrm{ZnO}$ materials showed poor device performance because $\mathrm{ZnO}$ nanowires that were significantly longer than the active layer thickness, and individual wires were bridging between the electrodes forming shorts. While the shorter $\mathrm{ZnO}$ nanowires should not be long enough to penetrate both electrodes, the increased surface conductivity from the conjugated TPP may be responsible for this increased leakage current. Regardless, it does not appear that the DDT functionalization is increasing the surface resistance of the $\mathrm{ZnO}$ structures. The high pass currents for TPP and DDT functionalized devices are close enough to each other to imply that resistance is not the issue for the DDT functionalized devices.

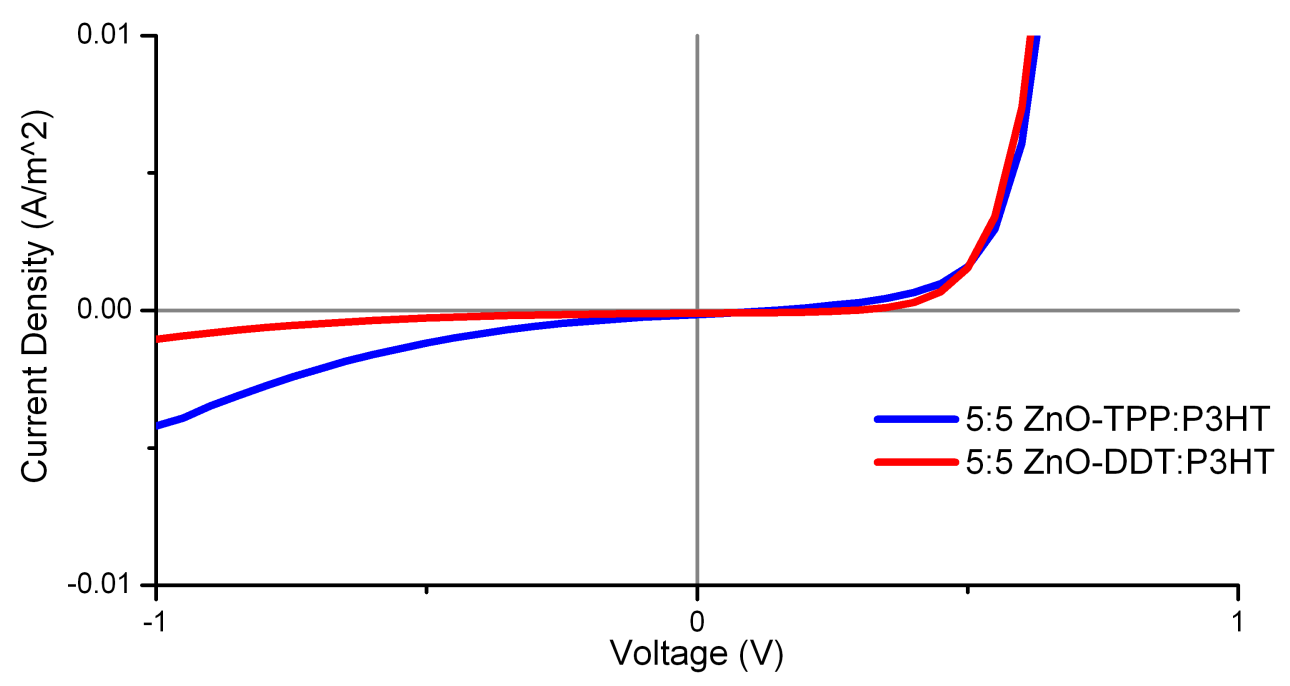

Figure 34 - Typical device dark curve from the 5:5 functionalized ZnO:P3HT groups

The final device efficiency data comes as little surprise, with the 5:5 ZnOTPP:P3HT devices scoring the highest power conversion efficiencies after the TPP-ZnO showed higher $\mathrm{V}_{\text {oc }}$ 's than the DDT-ZnO devices, and with the short 
circuit currents significantly higher in the 5:5 functionalized ZnO:P3HT devices than the 1:9 functionalized $\mathrm{ZnO}: \mathrm{P} 3 \mathrm{HT}$ devices. The one low performing device in the group also had the lowest $\mathrm{J}_{\mathrm{sc}}$, and a poor fill factor, see Figure 35 . There are many reasons that a single device can perform poorly, but being as the standard deviation of the efficiencies stayed small with the loss in performance, it is reasonable to say that the issue is device centric. It is likely that the issue came about during the active layer application, when the layer of polymer and $\mathrm{ZnO}$ may have been spun on too thin or too thick. It's also possible that the electrode was not applied as uniformly as it was in other devices; the device in question was placed on one of the outer edges of the aluminum evaporator for cathode application. While the 5:5 TPP-ZnO:P3HT group had the best performing devices, $0.03 \%$ power conversion efficiency is not the magnitude that these devices were hoped to produce. Previous work in this lab with the P3HT:PCBM system has yielded devices $>3 \%$ power conversion efficiencies.

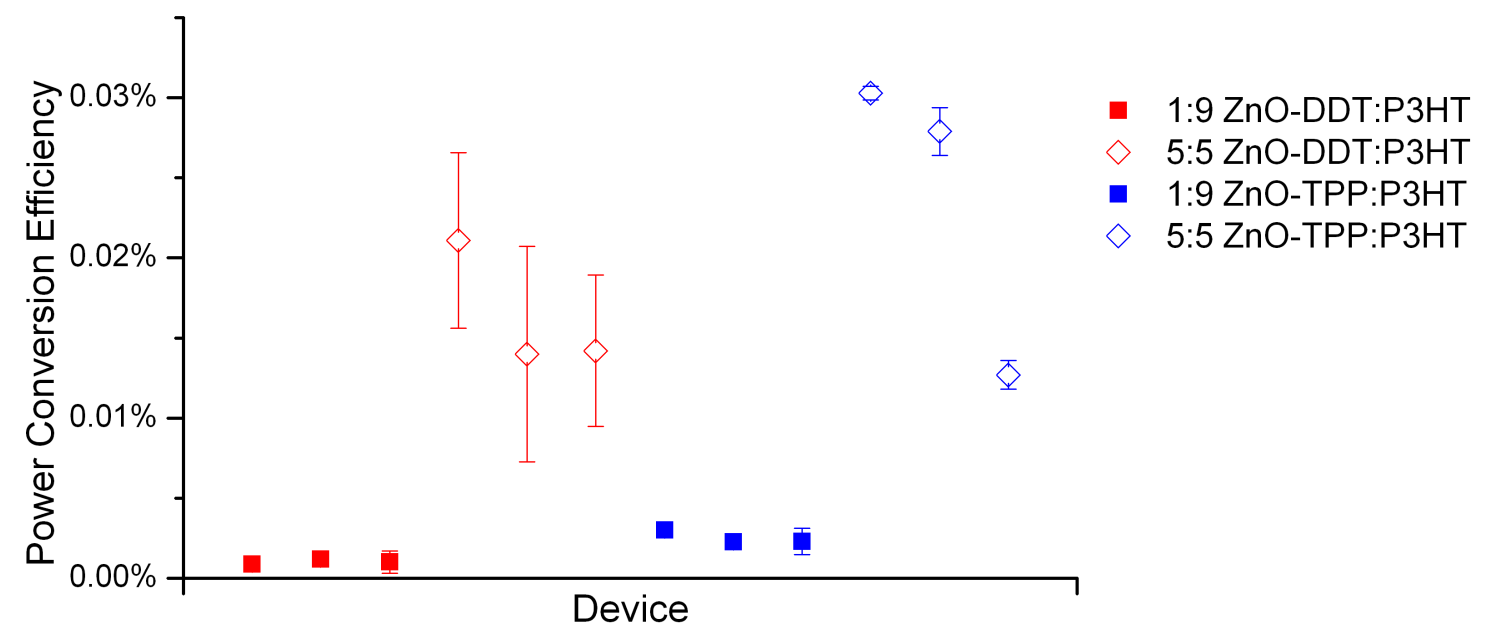

Figure 35 - Power Conversion efficiency plot of surface functionalized $\mathrm{ZnO}$ :P3HT bulk heterojunction hybrid devices 
Another important element to device fabrication is confirming that devices are absorbing enough light too effectively generate power. Measuring the solid state absorbance of these devices to obtain absorption spectra gives an easy check to that end, results of which are shown in Figure 36. Optical density shows that the 5:5 ZnO-DDT:P3HT and 5:5 ZnO-TPP P3HT devices had maximum single pass absorbance of $\sim 1$ optical density. Since the rear electrode of the solar cells are aluminum and have a near mirror finish, incident light has two passes through the active layer, so the 5:5 device group is absorbing $\sim 99 \%$ of incident light at the absorption peak. The 1:9 devices group, with it's lower absorbance of $\sim 0.5$ is still absorbing $90 \%$ of incident light at the absorption peak. This is more than enough light to create a well functioning solar cell.

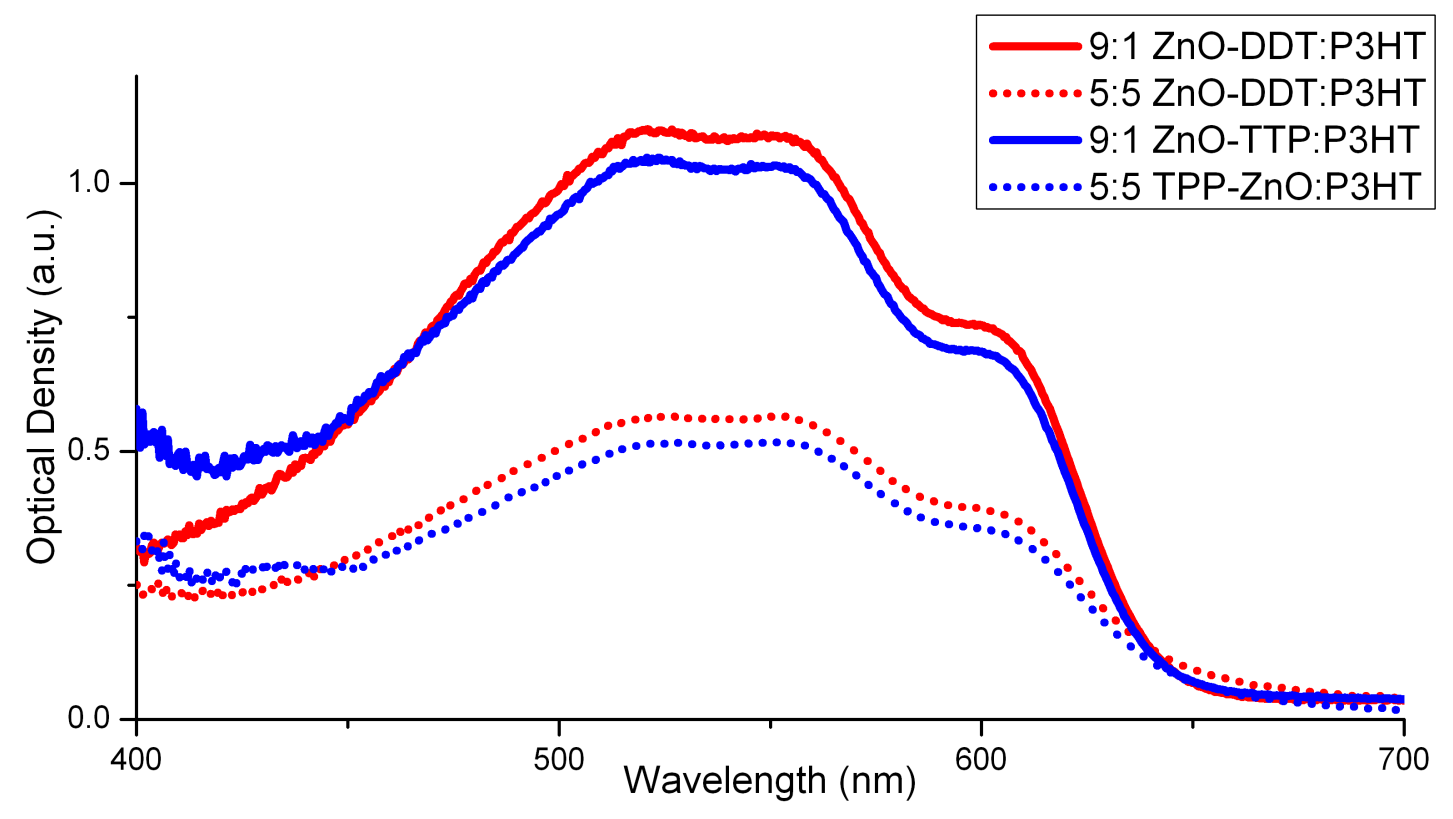

Figure 36 - Optical density measurements for solid film absorbances of surface functionalized $\mathrm{ZnO}$ OP3HT bulk heterojunction hybrid devices 
A fill factor in a well performing organic photovoltaic device is typically around 60 . For all devices tested the fill factor fell somewhere between 25 and 50, see Figure 37 . While these numbers are not particularly impressive, they are also not particularly low, which begs the question, what is causing the poor device performance.

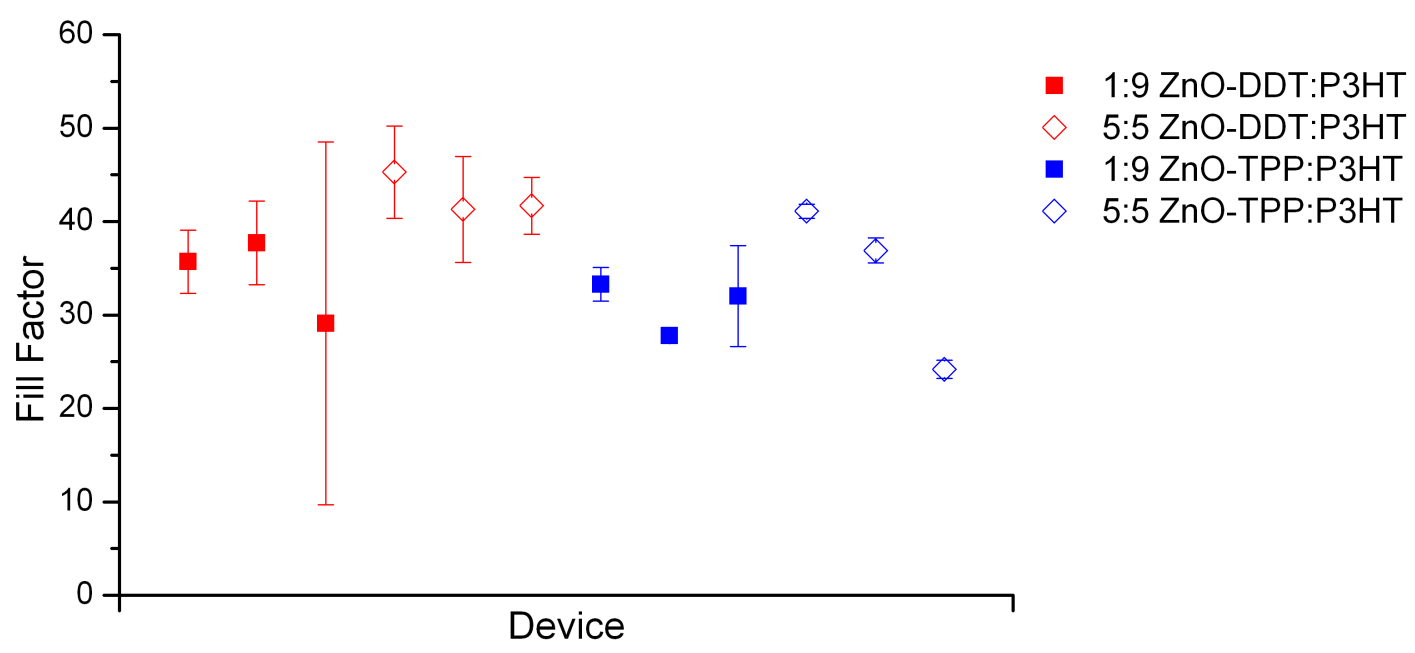

\section{Figure 37 - Fill factor plot of surface functionalized $\mathrm{ZnO}$ :P3HT bulk heterojunction hybrid devices}

Since fill factor is particularly sensitive to charge extraction losses, we can conclude that charge extraction is not the dominant loss mechanism in our synthesized devices. The data from the dark currents showed that leakage was prevalent in the ZnO-DDT:P3HT and ZnO-TPP:P3HT devices, but leakage of the amount that was observed would not explain the extremely low performance of these cells. Resistive losses are ruled out by the high forward pass currents, and thermalization losses could not be responsible because the $V_{o c}$ of these devices is comparable with previous $\mathrm{P} 3 \mathrm{HT}$ :PCBM devices with power conversion efficiencies that were orders of magnitude higher. The dominant loss mechanism 
that is left is recombination, implying that the polymer regions are too large. This matches well with the observed effect that larger polymer concentrations in the active layer made the device performance worse, as comparing the 5:5 and 1:9 functionalized $\mathrm{ZnO}: \mathrm{P} 3 \mathrm{HT}$ groups. Further corroboration on this conclusion is provided by the short circuit currents measured, where the devices with 5 times greater concentration of $\mathrm{ZnO}$ produced 10 times more short circuit current. Future work on this system will benefit from using smaller loadings of polymer, and will also likely gain performance if $\mathrm{ZnO}$ can be synthesized in even smaller nanowires than the current synthesis method. 


\section{CONCLUSIONS}

It has been shown that the $\mathrm{ZnO}: \mathrm{P} 3 \mathrm{HT}$ can be used to create functional solar devices via nanowire surface functionalization. The loss mechanisms for such devices have been identified as being dominated by recombination, but with acceptable charge extraction. Core shell nanostructures were also synthesized using carboxylated polythiophenes of side chain lengths ranging from three to six carbon alkyl chains. Linkage of the polymer to the $\mathrm{ZnO}$ nanowires via chemical grafting was confirmed, and the conformational order of the attached polymer was evaluated. It was determined that the core shell structure was a desirable system for continued solar cell fabrication and testing. 


\section{REFERENCES}

1. Jacobson, M. A.; Delucchi, M. A. A Path to Sustainable Energy by 2030. Scientific American, November 2009, pp 58-65.

2. Short-Term Energy Outlook (STEO); U.S. Energy Information Administration, May 2014

3. Pimentel, D.; Herz, M.; Glickstein, M.; Zimmerman, M.; Allen, R.; Becker, K.; Evans, J.; Hussain, B.; Ryan, R.; Grosfeld, A.; Seidel, T. Renewable Energy: Current and Potential Issues. BioScience. 2002, 52, 12, 1111-1120.

4. Office of Energy Efficiency and Renewable Energy. Sunshot: Mission. http:// energy.gov/eere/sunshot/mission (accessed May 29th, 2014).

5. Office of Energy Efficiency and Renewable Energy. Progress Report: Advancing Solar Energy Across America. http://www.energy.gov/articles/progress-reportadvancing-solar-energy-across-america (accessed May 29th, 2014)

6. Perlin, J. From Space to Earth: The Story of Solar Electricity, Harvard University Press, 1999.

7. Einstein, A. Über einen die Erzeugung und Verwandlung des Lichtes betreffenden heuristischen Gesichtspunkt. Annalen der Physik. 1905, 17, 6, 132-148.

8. Chapin, D. M.; Fuller, C. S.; Pearson, G. L.; A New Silicon p-n Junction Photocell for Converting Solar Radiation into Electrical Power. Journal of Applied Physics. 1954, $25,5,676-677$.

9. News Release: NREL Solar Cell Sets World Efficiency Record at 40.8\%; NR-2708; NREL, CO, August 13, 2008.

10. National Center for Photovoltaics. Research Cell Efficiency Chart. http:// www.nrel.gov/ncpv/images/efficiency chart.jpg (accessed May 29th, 2014)

11. Taylor, J. R.; Zafiratos, C. D.; Dubson, M. A. Modern Physics for Scientists and Engineers. 2nd ed.; Prentice Hall, Upper Saddle River, NJ, 2004.

12. Brown, R. F. Solid State Physics, An Introduction for Scientists and Engineers. El Corral Publications, San Luis Obispo, CA, 2006.

13. McGehee D.G.; Topinka M.A. Solar cells: Pictures from the blended zone. Nature Materials. 2006, 5 9, 675-676.

14. Pankove, J. I. Optical Processes in Semiconductors. Dover, Mineola, NY, 1971.

15. Shirakawa, H.; Louis, E. J.; MacDiarmid, A. G.; Chiang, C. K.; Heeger, A. J. Synthesis of electrically conducting organic polymers: Halogen derivatives of polyacetylene, $(\mathrm{CH})_{x}$. Journal of the Chemical Society, Chemical Communications. 1977, 16, 578-580.

16. Skotheim, T. A.; Elsenbaumer, R. L.; Reynolds, J. R. Handbook of Conducting Polymers. Marcel Dekker, Inc., New York, New York, 1998.

17. Isaacs, E. B.; Sharifzadeh, S.; Ma, B.; Neaton, J. B. Relating Trends in FirstPrinciples Electronic Structure and Open-Circuit Voltage in Organic Photovoltaics. J. Phys. Chem. Lett. 2011, 2, 2531-2537 
18. Bücher, K.; Bruns, J.; Wagemann, H.G.; Absorption coefficient of silicon: An assessment of measurements and the simulation of temperature variation. Journal of Applied Physics 1994, 75, 1127-1132

19. Kim, J.Y.; Lee, K.; Coates, N. E.; Moses, D.; Nguyen, T. Q.; Dante, M.; Heeger, A. J. Efficient Tandem Polymer Solar Cells Fabricated by All-Solution Processing. SCIENCE 2007, 317, 222-225

20. Brabec, C.; Dyakonov, V.; Scherf, U. Organic Photovoltaics: Materials, Device Physics, and Manufacturing Technologies; Wiley-VCH: Weinheim, Germany, 2008.

21. Zardetto, V.; Brown, T. M.; Reale, A.; Carlo, A. D. Substrates for Flexible Electronics: A Practical Investigation on the Electrical, Film Flexibility, Optical, Temperature, and Solvent Resistance Properties. Journal Of Polymer Science Part B: Polymer Physics 2011, 49, 638-648

22. Green, M. A.; Emery, K.; Hishikawa, Y.; Warta, W.; Dunlop, E. D. Solar cell efficiency tables. Prog. Photovolt: Res. Appl. 2012; 20, 12-20

23. Yang, T.; Cai, W.; Qin, D.; Wang, E.; Lan, L.; Gong, X.; Peng, J.; Cao, Y. SolutionProcessed Zinc Oxide Thin Film as a Buffer Layer for Polymer Solar Cells with an Inverted Device Structure. J. Phys. Chem. C. 2010, 114, 6849-6853

24. Shaw, P. S.; Ruseckas, A.; Samuel, I. D. W. Exciton Diffusion Measurements in Poly(3-hexylthiophene). Adv. Mater. 2008, 20, 3516-3520

25. Yang, X.; Loos, J.; Veenstra, S. C.; Verhees,W. J. H.; Wienk, M. M.; Kroon, J. M.; Michels, M. A. J.; Janssen, R. A. J. Nanoscale Morphology of High-Performance Polymer Solar Cells, Nano Letters, 2005 5, 4, 579-583

26. Peet, J.; Heeger, A. J.; Bazan, G. C. "Plastic" Solar Cells: Self-Assembly of Bulk Heterojunction Nanomaterials by Spontaneous Phase Separation. Accounts Of Chemical Research. 2009, 42, 11, 1700-1708

27. Shrotriya, V.; Li, G.; Yao, Y.; Moriarty, T.; Emery, K.; Yang, Y. Accurate Measurement and Characterization of Organic Solar Cells. Adv. Funct. Mater. 2006, 16, 20162023

28. Servaites, J. D.; Ratner, M. A.; Marks, T. J. Organic solar cells: A new look at traditional models. Energy Environ. Sci. 2011, 4, 4410-4422

29. Heremans, P.; Cheyns, D.; Rand, B. P. Strategies for Increasing the Efficiency of Heterojunction Organic Solar Cells: Material Selection and Device Architecture. Accounts Of Chemical Research. 2009, 42, 11, 1740-1747

30. Wong, W. Y.; Wang, X. Z.; He, Z.; Chan, K. K.; Djuris`ic', A. B.; Cheung, K. Y.; Yip, C. T.; Man-Ching, A.; Xi, Y. Y.; Mak, C. S. K.; Chan, W. K. Tuning the Absorption, Charge Transport Properties, and Solar Cell Efficiency with the Number of Thienyl Rings in Platinum-Containing Poly(aryleneethynylene)s. J. Am. Chem. Soc. 2007, $129,14372-14380$

31. Vandewal, K.; Gadisa, A.; Oosterbaan, W. D.; Bertho, S.; Banishoeib, F.; Severen, I. V.; Lutsen, L.; Cleij, T. J.; Vanderzande, D.; Manca, J. V. The Relation Between Open-Circuit Voltage and the Onset of Photocurrent Generation by Charge-Transfer Absorption in Polymer : Fullerene Bulk Heterojunction Solar Cells. Adv. Funct. Mater. 2008,18, 2064-2070 
32. Brown, P. J.; Thomas, D. S.; Köhler, A.; Wilson, J. S.; Kim, J. S.; Ramsdale, C. M.; Sirringhaus, H.; Friend, R. H. Effect of interchain interactions on the absorption and emission of poly 3-hexylthiophene. Physical Review B. 2003, 67, 064203

33. Sirringhaus, H.; Brown, P. J.; Friend, R. H.; Nielsen, M. M.; Bechgaard, K.; Langeveld-Voss, B. M. W.; Spiering, A. J. H.; Janssen, R. A. J.; Meijer, E. W.; Herwig, P.; de Leeuw, D. M. Two-dimensional charge transport in self-organized, highmobility conjugated polymers. Nature. 1999, 401, 685-688

34. Yang, T.; Cai, W.; Qin, W.; Wang, E.; Lan,L.; Gong, X.; Peng, J.; Cao, Y. SolutionProcessed Zinc Oxide Thin Film as a Buffer Layer for Polymer Solar Cells with an Inverted Device Structure. J. Phys. Chem. C, 2010, 114, 14, 6849-6853

35. Schmidt-Mende, L.; MacManus-Driscoll, J. L. ZnO - nanostructures, defects, and devices. MaterialsToday, 2007, 10, 5, 40-48

36. Wang, H.; He, Y.; Li, Y.; Su, H. Photophysical and Electronic Properties of Five PCBM-like $\mathrm{C}_{60}$ Derivatives: Spectral and Quantum Chemical View. J. Phys. Chem. A. 2012, 116, 255-262

37. Zhao, L.; Wang, J.; Lin, Z. Semiconducting nanocrystals, conjugated polymers, and conjugated polymer/nanocrystal nanohybrids and their usage in solar cells. Frontiers of Chemistry in China. 2010, 5, 1, 33-34

38. Allen, C. G. B., D. J.; Brenner, T. M.; Weigand, C. C.; Albin, J. M.; Steirer, K. X.; Olson, D. C.; Ladam, C.; Ginley, D. S.; Collins, R. T.; Furtak, T. E. Alkyl Surface Treatments of Planar Zinc Oxide in Hybrid Organic/Inorganic Solar Cells. Journal of Physical Chemistry C. 2012, 116, 8872-8880

39. Park, B. L.; Jung-Hyun; Chang, M.; Reichmanis, E. Exciton Dissociation and Charge Transport Properties at a Modified Donor/Acceptor Interface: Poly(3-hexylthiophene)/ Thiol-ZnO Bulk Heterojunction Interfaces. Journal of Physical Chemistry C. 2012, 116, 4252-4258.

40. Monson, T. C. L.; Matthew T.; Olson, D. C.; Lee, Y.; Hsu, J. W. P. Photocurrent Enhancement in Polythiophene- and Alkanethiol-Modified ZnO Solar Cells. Advanced Materials. 2008, 20, 4755-4759

41. Rakshit, T. M., S. P.; Manna, I.; Ray, S. K. CdS-Decorated ZnO Nanorod Heterostructures for Improved Hybrid Photovoltaic Devices. Applied Materials \& Interfaces. 2012

42. Zhang, S. M., Pawel W.; Keskar, G.; Pfefferle, L. D.; Osuji, C. O. Lyotropic SelfAssembly of High-Aspect-Ratio Semiconductor Nanowires of Single-Crystal ZnO. Langmuir, 2011, 27, 11616-11621

43. Zhang, B. K., Tao; Xu, W.; Su, R.; Gao, Y.; Cheng, G. Surface Functionalization of Zinc Oxide by Carboxyalkylphosphonic Acid Self-Assembled Monolayers. Langmuir 2010, 26, 6, 4514-4522.

44. Allen, C. G. B., D. J.; Albin, J. M.; Oertli, H. E.; Gillaspie, D. T.; Olson D. C.; Furtak, T. E.; Collins, R. T. Surface Modification of ZnO Using Triethoxysilane-Based Molecules. Langmuir, 2008, 24, 13393-13398.

45. Park, Y. S.; Schneider, J. R.; Index of Refraction of ZnO. J. Appl. Phys. 1968, 39, 7, 3049-3052 
46. Worfolk, B. J.; Rider, D. A.; Elias, A. L.; Thomas, M.; Harris, K. D.; Buriak, J. M. Bulk Heterojunction Organic Photovoltaics Based on Carboxylated Polythiophenes and PCBM on Glass and Plastic Substrates. Adv. Funct. Mater. 2011, 21, 1816-1826

47. Vaynzof, Y.; Kabra, D.; Zhao, L.; Chua, L. L.; Steiner, U.; Friend, R. H. SurfaceDirected Spinodal Decomposition in Poly[3-hexylthiophene] and C61-Butyric Acid Methyl Ester Blends. ACS Nano. 2011, 5, 1, 329-336

48. Jørgensen, M.; Norrman, K.; Krebs, F. K. Stability/degradation of polymer solar cells. Solar Energy Materials \& Solar Cells. 2008, 92, 686-714 